\title{
Nanochips assisted peptide screening for clinical development of CAR-T cell immunotherapy
}

Trang Anh Nguyen-Le

Helmholtz-Zentrum Dresden-Rossendorf

Tabea Bartsch

Helmholtz-Zentrum Dresden-Rossendorf

Robert Wodtke

Helmholtz-Zentrum Dresden-Rossendorf

Florian Brandt

Helmholtz-Zentrum Dresden-Rossendorf

Claudia Arndt

Helmholtz-Zentrum Dresden Rossendorf (HZDR)

Anja Feldmann

Helmholtz-Zentrum Dresden Rossendorf (HZDR)

Diana Isabel Sandoval Bojorquez

Helmholtz-Zentrum Dresden-Rossendorf

Arnau Perez Roig

Helmholtz-Zentrum Dresden-Rossendorf

Bergoi Ibarlucea

Dresden University of Technology https://orcid.org/0000-0002-9899-1409

\section{Seungho Lee}

POSTECH

Chang-Ki Baek

POSTECH https://orcid.org/0000-0002-2852-6683

\section{Gianaurelio Cuniberti}

Technical University (TU) Dresden https://orcid.org/0000-0002-6574-7848

\section{Ralf Bergmann}

Helmholtz Zentrum Dresden Rossendorf

\section{Edinson Puentes-Cala}

Corporación para la Investigación de la Corrosión (CIC)

Javier Andrés Soto

Universidad de Santander

\section{Biji T. Kurien}

University of Oklahoma Health Sciences Center

Michael Bachmann 
Helmholtz-Zentrum Dresden-Rossendorf (HZDR)

Larysa Baraban ( $\sim$ l.baraban@hzdr.de)

Helmholtz-Zentrum Dresden-Rossendorf https://orcid.org/0000-0003-1010-2791

\section{Article}

Keywords: CAR-T cells, UniCAR-T cells, cancer, silicon nanowires, nanosensor, field effect transistor, ELISA, immunotherapy

Posted Date: August 26th, 2021

DOl: https://doi.org/10.21203/rs.3.rs-841987/v1

License: (c) (i) This work is licensed under a Creative Commons Attribution 4.0 International License. Read Full License 


\section{Nanochips assisted peptide screening for clinical development of CAR-T cell immunotherapy}

Trang Anh Nguyen-Le ${ }^{1 \ddagger}$, Tabea Bartsch $^{1 \ddagger}$, Robert Wodtke ${ }^{1}$, Florian Brandt ${ }^{1,2}$, Claudia Arndt $^{1,3}$, Anja Feldmann ${ }^{1 *}$, Diana Isabel Sandoval Bojorquez ${ }^{1}$, Arnau Perez Roig ${ }^{1}$, Bergoi Ibarlucea ${ }^{4}$, Seungho Lee ${ }^{5}$, Chan-Ki Baek ${ }^{5}$, Gianaurelio Cuniberti ${ }^{4}$, Ralf Bergmann ${ }^{1,6}$, Edinson

Puentes-Cala ${ }^{7}$, Javier Andrés Soto ${ }^{8}$, Biji T. Kurien ${ }^{9}$, Michael Bachmann ${ }^{1,10-13 *}$, Larysa Baraban $^{1 *}$

${ }^{1}$ Institute of Radiopharmaceutical Cancer Research, Helmholtz-Zentrum Dresden-Rossendorf e. V. (HZDR), 01328 Dresden, Germany

2 Fakultät Chemie und Lebensmittelchemie, Technische Universität Dresden, Mommsenstraße 4, 01062 Dresden, Germany

${ }^{3}$ Mildred Scheel Early Career Center, Faculty of Medicine Carl Gustav Carus, TU Dresden, 01307 Dresden, Germany

${ }^{4}$ Institute for Materials Science, Max Bergmann Center for Biomaterials, Center for advancing electronics Dresden (cfaed), Technische Universität Dresden, 01069 Dresden, Germany

${ }^{5}$ Department of Convergence IT Engineering, Pohang University of Science and Technology, Pohang 37673, Republic of Korea

6 Department of Biophysics and Radiation Biology, Semmelweis University, Budapest, Hungary.

${ }^{7}$ Corporación para la Investigación de la Corrosión (CIC), Piedecuesta 681011, Colombia

${ }^{8}$ Universidad de Santander, Cúcuta, Colombia

9 The Arthritis and Clinical Immunology Program, Oklahoma Medical Research Foundation and University of Oklahoma Health Sciences Center, Oklahoma City, OK 73104, USA; BijiKurien@omrf.org (B.T.K.)

${ }^{10}$ Tumor Immunology, University Cancer Center (UCC), University Hospital Carl Gustav Carus Dresden, Technische Universität Dresden, 01307 Dresden, Germany.

${ }^{11}$ National Center for Tumor Diseases (NCT), Dresden, Germany. Faculty of Medicine and University Hospital Carl Gustav Carus, Technische Universität Dresden, 01307 Dresden, Germany.

${ }^{12}$ German Cancer Research Center (DKFZ), Heidelberg, Germany.

${ }^{13}$ German Cancer Consortium (DKTK), Dresden, Germany.

*Corresponding author: I.baraban@hzdr.de, m.bachmann@hzdr.de

‡ These authors made an equal contribution to this work 


\section{ABSTRACT:}

Immunotherapy using CAR-T cells is a new paradigm technology for cancer treatment. To avoid severe side effects and tumor escape variants observed for conventional CAR-T cells approach, adaptor CAR technologies are under development, where intermediate target modules redirect immune cells against cancer cells. In this work, silicon nanowire field effect transistors are used to assist in the development of target modules for an optimized CAR-T cell operation. Focusing on a library of seven variants of E5B9 peptide that is used as CAR peptide epitope, we performed multiplexed binding tests in serum using nanosensor chips. Peptides have been immobilized onto the sensor to compare the signals of transistor upon titration with anti-E5B9 antibodies. Correlation analysis of binding affinities and sensitivities enabled a selection of best candidates for the interaction between CAR and target modules. Finally, cytotoxic functionality of CAR-T cells in combination with the selected target modules were successfully proven. Our results open the perspective for the nanobiosensorics to go beyond the early diagnostics in the field of clinical cancer research, and paves the way towards personalization and efficient monitoring of the immunotherapeutic treatment, where the quantitative analysis with the standard techniques is not an option.

Key words: CAR-T cells, UniCAR-T cells, cancer, silicon nanowires, nanosensor, field effect transistor, ELISA, immunotherapy 


\section{INTRODUCTION}

Cancer affects our society globally. According to the most recent cancer statistics [1], approximately 19 million new cases of cancer and around 10 million deaths were documented in 2020. Surgery, radiotherapy and chemotherapy are the most common types of cancer treatments available nowadays [2]. In recent years, immunotherapy, especially $\mathrm{T}$ cells genetically modified with chimeric antigen receptor (CARs) are considered as milestone technology for cancer treatment [3,4]. After remarkable remissions observed in clinical trial patients, anti-CD19 and anti-CD269 CAR-T cells received approval from Health Canada and U.S Food and Drug Administration (FDA) [5] for treatment of hematologic malignancies in 2017. Genetically modified CAR-T cells express specific receptors (CARs) on their surface for an effective tumor addressing. Upon binding on tumor cells, CARs induce a signal cascade resulting in $T$ cell activation and thus elimination of cancer cells. Despite the demonstrated clinical success, CAR-T cell therapy can be accompanied by severe side effects including cytokine release syndrome, neurologic toxicity, on-target/off-tumor reactions, and anaphylaxis [6-8].

While reactivity of conventional CAR-T cells cannot be controlled in the human body, the recently established adaptor universal (Uni)CAR platform enables the on/off switch of CAR-T cell activity providing an improved safety management [9-13]. This system consists of two components: (I) T cells expressing UniCARs, that comprise the extracellular anti-E5B9 singlechain fragment variable (scFv) derived from the anti-E5B9 monoclonal antibody (mAb), the transmembrane domain and intracellular signaling domains, and (II) target modules (TMs) that consist of an antigen binding moiety equipped with the peptide epitope E5B9 (Figure 1A). TMs play a very important role in this concept as they simultaneously bind to tumor antigens and the UniCAR-T cell and mediate tumor lysis. As the TMs have usually a short biological half-life [14], the UniCARs will, thus, switch off after stopping the TM infusion. This opens an instrument of a control over the therapy, which is absent in the conventional CAR therapy approach.

Note, the strength and persistence of the interaction between UniCAR-T cells and tumor cells is of paramount importance and its optimization is valuable for an advanced therapeutic outcome. It depends on the recognition of the E5B9 peptide epitope (as part of the TM) by the extracellular UniCAR anti-E5B9 scFv. Furthermore, as the UniCAR platform is subject of current clinical trials (NCT04633148, NCT04230265 [15]), the optimal dosing of TMs and UniCAR-T cells in human blood are also of high interest. Despite responding to therapy, first clinical trial patients showed very low concentrations of TMs in the blood that could so far not be detected using conventional techniques [15]. Taking into account the potentially high amount of molecular complexes to be tested, and the low concentrations expected in blood 
post-injections, the use of compact tools capable of multiplexed detection with exceptional sensitivity is indispensable for such studies.

Nanobioelectronics is an emerging field considered for future clinical applications, offering highly sensitive, label-free, rapid and reagent-saving analytical tools. As a part of this field nanobiosensors, have shown their efficiency in ultrasensitive detection of low concentrations of biomolecules [16-26] and single cells [27-30]. While the major focuses in the community are dedicated to the delivery and characterization of new efficient transducer materials for pointof-care biosensors [31-34], there is a limited number of reports with the clinical applications of such nanodevices [27, 35-40]. We attribute it to the necessary complexity of the system, need for a long-term process monitoring, and thus, a stable performance of the sensor. Particularly, when reviewing the application of nanobiosensors for cancer research, the major contributions have been done mainly in the early diagnosis of cancer with more than $70 \%$ of articles published in the last decade (see Figure 1B). Next $20-25 \%$ of works included the monitoring of the patient response to the treatment through exhaled breath [41], various cell signaling agents [42-44], and growth factors [45, 46]; counting circulating cancer cells [47-49]; detecting mutation in cancer [50-53]; tracking cancer progression [54, 55]; and quantifying anti-cancer drug residual in patient $[56,57]$. Meanwhile, application of nanoelectronics in the development of the cancer therapy remains unexplored with only a handful of quality works and lack of breakthrough proof-of-concept. Works in this direction largely demonstrate monitoring of living cancer cell response for e.g. drug screening or change in treatment condition [58-61] and detection of drug-resistance gene/cell [62, 63] [64]. Still, there is confidence that the nanosensors can accelerate the progress of many clinical applications, beyond traditionally considered diagnostics and point-of-care. Thus, works related to the use of nanobioelectronics for the development of personalized cancer therapies and monitoring of therapy efficiency are still expected. To the best of our knowledge, the utilization of a purely nanoelectronic biosensor in immunotherapy is completely absent.

In this work, we go beyond the traditional use of the nanobiosensors in the diagnostics area and explore their ability to assist in clinical development and optimization of the UniCAR-T cell therapy, at a proof-of-concept level. For these purposes, we employ an array of top down fabricated silicon nanowire (SiNW) ion-sensitive field-effect transistors (ISFET) - responding to changes in the distribution of the surface charge within Debye layer, mainly affecting the charge carriers in the conductance channel [64]. SiNW based devices have outstanding relevance for this specific task due to several arguments: (i) their long term stability as biosensor device even upon multiple use, (ii) lower stand-by energy consumption, and (iii) compatibility to a technology based on complementary metal oxide semiconductor (CMOS) and thus higher commercialization potential [65]. Although the use of SiNW FETs as 
biosensors have been reported for more than a decade ago $[66,67]$, their applications still did not enter the clinics.

In the following, we applied the nanoscopic FET sensor to analyze the interaction between the peptide epitope E5B9 and the anti-E5B9 scFv, used for optimization of the UniCAR approach. Success at this stage creates a tool for detection of UniCAR-T cells or TMs in the blood or sera of patients, receiving UniCAR-T cell therapy. This aspect is of high interest for continuous monitoring of the UniCAR approach efficiency.

To evaluate the SiNW FET sensor-based technology and to identify the most suitable E5B9 peptide to redirect UniCAR-T cells, the interaction between seven E5B9 peptide variants (Figure 1C) and the corresponding anti-E5B9 mAb was investigated using the SiNW FET sensors in comparison to ELISA. Therefore, the multiple peptides have been immobilized onto the sensing area (Figure 1D), and threshold voltage shift of the transistor was recorded upon titration of anti-E5B9 mAb in order to pre-select the optimal epitope for UniCAR-T cell redirection. Finally, in vitro cytotoxicity models were used to test whether TMs equipped with different E5B9 variants are able to redirect UniCAR-T cells for elimination of prostate stem cell antigen (PSCA)-expressing tumor cells [77].

\section{RESULTS AND DISCUSSION}

\subsection{Fabrication of nanowire based FET sensors}

A sensor chip consisting of $16 n$-doped $\left(5 \times 10^{18} \mathrm{~cm}^{-3}\right.$ phosphorous) SiNW FET devices (Figure 2A) was fabricated on an 8-inch silicon-on-insulator (SOI) wafer, as described in previous works [16, 17, 68-71] and Materials and Methods (Figure 2A left panel). The width of a single wire is about $50 \mathrm{~nm}$; assembly into a honeycomb pattern results in the total sensing area of about $45 \mu \mathrm{m}^{2}$. Compared to an array of straight wires, the patterned assembly of nanowires (Figure 2A right panel), offers large sensing area, better electrical characteristics, and higher mechanical stability, making it preferable for biosensing applications [70, 72, 73]. Nanowires and reference electrode are directly exposed to the environment, while the remaining elements of the chip are isolated using SU-8 photoresist. During measurement, a liquid is placed in the contact with the nanowires, covering the reference electrode and the SiNW channel. Attachment of the biomolecules to the surface of the silicon changes the surface potential at the nanowires, thus, influencing the conductance in the nanowire structure [74-76]. For the case of $n$-typed silicon channel an increase in conductance is expected with positive gate voltage bias, while a decrease is anticipated with negative gate voltage as electrons are the main carriers in $\mathrm{n}$-doped silicon. Figures $2 \mathrm{~B}$ show typical transfer characteristics of the SiNW FET measured in deionized water. The source-drain current $\left(\mathrm{I}_{\mathrm{SD}}\right)$ increases when rising the 
source-drain voltage $\left(\mathrm{V}_{\mathrm{SD}}\right)$ with different gate bias $\left(\mathrm{V}_{\mathrm{G}}\right)$. In addition, the source-drain current also escalated as higher positive gate voltage is applied which clearly indicates n-type behavior of SiNW FET. Due to heavily doped channel with phosphorus, inversion mode of $n$-type FET has been converted to accumulation mode. As a result, the device is normally in an ON-state at the gate bias $\mathrm{V}_{\mathrm{G}}=0 \mathrm{~V}$ (Figure $2 \mathrm{~B}$ ), which is advantageous for low power sensor application.

\subsection{Functionalization of the silicon surface}

Figure 1A represents a scheme of the UniCAR approach: genetically modified UniCAR-T cells interact with the tumor cells via TMs [77] [78, 79]. TMs consist of two components: the peptide epitope E5B9 (red sphere) recognized by UniCAR-T cells and a binding domain that binds to a certain target antigen overexpressed on the surface of tumor cells (orange triangles). The interaction between effector and target cells depends on the affinity of the UniCAR anti-E5B9 scFv towards the peptide epitope E5B9 as part of the TM and can influence the functionality of the UniCAR system. Even a minor change in the amino acid sequence can, in principle, cause a reduction or increase of binding ability and thus, affect the efficiency of the UniCAR-T cell-based therapy. Therefore, a fast and simple screening method for evaluation of the peptide recognition could be valuable to identify the optimal peptide sequence allowing a well-balanced interaction between UniCAR-T cells and TMs resulting in efficient UniCAR-T cell activation and tumor cell lysis. In this study, we have generated different E5B9 peptide epitope variants and analyzed them via the SiNW FET technology.

Here, the SiNW sensors mimic the interaction of the peptide epitope E5B9 with the antiE5B9 $\mathrm{mAb}$ used for construction of the UniCAR to explore how alterations in E5B9 structure may influence the efficiency of the UniCAR system. Seven different variants of the peptide E5B9 were covalently bound to the SiNW surface (see Materials and Methods, and Figure 1C, D). The original sequence of E5B9 derived from the human La/SS-B protein includes ten amino acids (KPLPEVTDEY)[80], as highlighted in black in Figure 1C. In this work, we investigated two groups of E5B9 variants namely $M$ and A group. The M-peptides (M1, M2, M3) have one or two exchanged amino acids in the main sequence while the A-peptides (A1, A2) have additional functional groups at the $\mathrm{N}$ - or $\mathrm{C}$-terminus which might be useful for modification with chelators [81]. In addition, $\mathbf{M 0}$ and $\mathbf{A} \mathbf{0}$ were synthesized to resemble the wildtype E5B9 sequence and act as a reference in each group.

The process of covalently attaching these peptides to SiNW consists of three steps [82, 83] and is briefly described in Figure 2D (step 1 to 3) followed by a blocking step and anti-E5B9 $\mathrm{mAb}$ detection. Each step is verified by several methods including sessile drop contact angle measurement (Figure 2E), fluorescent microscopy (Figure 2F) and FET electrical measurements of the subthreshold voltage shift (Figure 2G). Briefly, the silicon nanowire chips were first treated with oxygen plasma and left in 3-triethoxysilylpropyl succinic anhydride 
(TESPSA) vapor for four hours as described in [82]. Measuring the transfer curve of SiNW FET, we observed a respective 'positive' shift of about $\sim 110 \mathrm{mV}$ compared to the bare sensor (Figure $2 \mathrm{G}$ ), indicating a change in surface potential at the nanowires. Next, we attached the peptide sequences at the silicon nanowire, incubating a drop of $0.1 \mathrm{mg} / \mathrm{mL}$ peptide solution in PBS, at room temperature. Peptides are expected to covalently bind to the SiNW surface after one hour in contact, due to a ring opening reaction forming an amide bond [33, 82, 84]. In this context, all peptides bear in addition to the free $\mathrm{N}$-terminus another primary amino group at the side-chain of the lysine residue. Even though the $\mathrm{p} K_{\mathrm{a}}$ value of $\varepsilon$-amino group might be higher than that of the $\alpha$-amino group [83], thus favouring the reaction via the $\alpha$-amino group at $\mathrm{pH}$ 7.4, amide bond formation probably occurs via both amino groups [84]. We then removed any unbound peptides by washing the sensor with PBS solution containing $0.05 \%$ TWEEN 20 and blocked remaining binding sites of TESPSA linker by ethanolamine. The contact angle results (Figure 2E) and fluorescent staining using anti-E5B9 mAbs and fluorescent-labeled-secondary Abs (Figure 2F) demonstrated the successful functionalization of the silicon surface $(\mathbf{S I} \mathbf{2 , 3})$.

\subsection{Detection of anti-E5B9 mAb binding using SiNW FET devices}

Next, we studied the response of each sensor to the presence of the anti- E5B9 mAb in PBS. A serial dilution of $m A b$ was prepared in $1 x$ PBS $(0.01 \mathrm{M}, \mathrm{pH}=7.4)$ ranging from $0.1 \mathrm{fg} / \mathrm{mL}$ to $0.1 \mathrm{ng} / \mathrm{mL}$. For each measurement, a $10 \mu \mathrm{L}$-drop of $\mathrm{mAb}$ was incubated on functionalized devices for 15 minutes. In a ISFET biosensor, the biological sensing activities must take place within Debye length so that the newly established electric field does not screen out by moving charge carriers in high ionic solution [33]. Typical physiological samples such as PBS and blood or serum result in a Debye length $\lambda_{D}$ of around $0.7 \mathrm{~nm}$, which is much shorter than the size of regular biomolecules (5-10 nm for $\mathrm{l}_{\mathrm{gG}}$ antibody) [85]. The incubation for Ab binding was made in 1x PBS to maintain the peptide's function, however, all electrical measurements were conducted in 0.01x PBS in which the Debye length $\lambda_{D}$ is $7.61 \mathrm{~nm}$ [86], sufficient to sense the binding of the mAb to the immobilized peptides (original E5B9 sequence with molecule weight $\sim 1.3 \mathrm{kDa}$ results in size $<1 \mathrm{~nm}$ [87]). Figure 3B exemplarily shows the transfer characteristics $\left(\mathrm{I}_{\mathrm{SD}}-\mathrm{V}_{\mathrm{G}}\right)$ of $\mathbf{A} \mathbf{0}$-functionalized SiNW FET after incubation with different concentrations of antiE5B9 mAb. The binding of charged species to the surface of the nanowire acts similar to applying a gate potential, modulating the conductance through the SiNW via depletion of enriching the charge carriers in the silicon channels. Note that all measurements were recorded continuously until steady state is reached and no drifting was observed afterwards, thus, the transfer curve shift is purely a contribution of anti-E5B9 mAb binding. After repetitive measurements of all peptides (each point is repeated 5 times), we observed no change in the slope of transfer characteristics but only the shift of the transfer characteristics. Therefore, we extract the threshold gate voltage $\left(\mathrm{V}_{\mathrm{T}}\right)$ at a fixed current through the source and drain $\mathrm{I}_{\mathrm{SD}}=10^{-}$ 
${ }^{7} \mathrm{~A}$, which can be translated as shift of transfer curve, to construct the calibration for further analysis (Figure $3 \mathrm{~A}$ ). Note that the $\mathrm{V}_{\mathrm{T}}$ change is detectable even at a concentration as low as one $\mathrm{fg} / \mathrm{mL}$. For better comparison between different peptide variants, Figure $3 \mathrm{~A}$ brings all calibration curves to the same origin in the same scale. The graph shows that the $V_{T}$ change of all variants follows a general binding assay curve in which the binding is quickly saturated after one or two orders of magnitudes. Nevertheless, the sensitivity between the peptides is significantly dissimilar (Figure 3C), because all peptides have distinct affinities to the same mAb, which is discussed more carefully below. In summary, current results show that functionalized SiNW sensors have potential for studying interaction between different modified E5B9 peptides and anti-E5B9 mAb.

\subsection{Binding of anti-E5B9 mAb to E5B9 peptides: SiNW FET versus ELISA}

We further confirm the binding data of the different E5B9 peptides to mAb obtained by SiNW FET sensors and compare these measurements with a traditional enzyme-linked immunosorbent assay (ELISA) (Figure 3A for FET and SI $\mathbf{4}$ for ELISA). While the reactivity of the biomolecules is directly observed by the change in electrical signal in the SiNW FET sensor, it is traced by an optical signal with addition of a labeled secondary Ab in ELISA (Material and Methods). Note that the working concentration used for testing in SiNW FET are five orders of magnitude lower than that in ELISA. In Figure $3 \mathrm{C}$ and Table 1, a comparison of performance parameters between the nanosensor and ELISA approaches is presented for all peptides, where we evaluate sensitivity, working range and limit of detection (LOD) for each test. Knowing that the anti-5B9 mAb is a typical immunoglobulin (Ig)G Ab with a molecular weight of approximately $150,000 \mathrm{~g} / \mathrm{mol}$, the detection limit of SiNW FET can go down to a few attomolar levels $\left(10^{-17} \mathrm{M}\right.$ ) (Figure $3 \mathrm{C}$ lower panel, Table 1) for most of the peptides, except for M3. In contrast, ELISA typically has a detection limit in the picomolar level $\left(10^{-12} \mathrm{M}\right)$. Overall, these data emphasize the superiority of SiNW FET over ELISA in terms of LOD and analyte consumption for applications where the detection of low levels of the analyte is necessary. Exemplarily, plasma levels of UniCAR TMs were below the limit of detection of the applied assay in patients participating in the ongoing clinical phase I trial of the UniCAR system, which showed very promising responses [15]. In this regard, for the detection of low concentrated TMs in patients the high sensitivity of the sensor can be beneficial or even required. The possibility to detect low concentrated TMs using the sensitive sensor technology paves the way towards future therapy monitoring.

Nanosensor technology can also play a role in treatment development. Regarding to peptide screening application, dissimilarity in nanosensor's response to the same target anti-E5B9 mAb reflecting different binding activity of the seven peptides, can help us to evaluate their suitability for TM construction. Binding studies between biomolecules are usually conducted 
through titration of one molecule to its counterpart until the equilibrium state is reached and is quantified by the dissociation constant $\left(K_{d}\right)$, or concentration that give half-maximum signal in ELISA. Pearson correlation analysis shows that the sensitivity of SiNW FET give better correlation to $K_{d}$ and other parameters obtained from ELISA (SI 5). Therefore, we believe that the sensitivity extracted from the steepest slope of the binding curve are representative to demonstrate binding capability of the mAb to the peptides in case of SiNW FET.

In this study, we exchange one or two glutamic acid(s) in the E5B9-sequence of $\mathrm{M}$-peptides or add a functional group in A-peptides to probe the alteration in the affinity of the anti-E5B9 $\mathrm{mAb}$ to the peptide variants. According to the sensitivity results in Figure $3 \mathrm{C}$, we expect higher affinities of the mAb to the M0 and $\mathbf{M} 1$ peptides than to the other mutated E5B9 variants (M2, M3). Thus, exchange of only one amino acid (M1, D to G) (Figure 1B) does not significantly alter binding ability of the anti-E5B9 mAb towards its epitope. However, replacement of two amino acids (M2, M3) reduced the sensitivity significantly showing that these amino acids are critical for the paratope-epitope interactions. On the other hand, the sensitivity of $\mathbf{A} 1$ and $\mathbf{A 2}$ are comparable to $\mathbf{A} \mathbf{0}$, which suggests that attachment of different functional groups does not significantly change the binding efficiency of the peptide. These are very useful information during the construction of TMs for UniCAR platform, which can now be provided using nanosensor.

\subsection{Influence of human blood serum on SiNW FET}

The concept of UniCAR-T cell therapy comprises that autologous T cells are isolated from the blood of patients, genetically modified ex vivo with the UniCARs and after their expansion adoptively transferred back into the patient. The TMs are administered by permanent intravenous infusion over several weeks to maintain effective concentrations of these rapidly eliminated molecules. To better monitor therapeutic effects but also possible side effects, it is of high interest to evaluate both UniCAR-T cell and TMs concentration in patient's blood during the treatment. Keeping in mind that TM-UniCAR interactions under physiological conditions will take place in human blood, we challenged our nanowire-based FET technique with the reactivity of TM elements on SiNW FET and anti-E5B9 mAb in human blood serum. For the proof-of-concept, we perform the study in the M group with mutant variants (M0, M1, M2 and M3) since this group of peptides includes the best and least-sensitive peptides and has the strongest correlation between ELISA and SiNW FET assays (Figure S5 B).

Blood serum contains many proteins, which in turn considerably change the background signal (Figure S6). Although, signal contribution from anti-E5B9 mAb is easily distinguishable (Figure S6), the high concentration of albumin protein in blood serum [88] might have negative effect on sensing response (Figure S7). While removing the unwanted protein is not an option (Figure S8), highly sensitive nanosensors allow us to conduct all further experiments in 10-time-diluted 
serum $(0.1 \mathrm{x}$ serum) where less variation in anti-E5B9 mAb detection is observed but still work in a much lower concentration range compare to conventional technique. All calibration curves are constructed by subtracting the measured signal to the signal of blank serum sample. The results are summarized in Figure 4. The results of $\mathbf{M 0}, \mathbf{M} \mathbf{1}$ and $\mathbf{M} \mathbf{3}$ in human serum is comparable to those in PBS (Figure 4A-B), e.g. M0 and M1 still give the best sensitivity, while M3 reveals weak binding, leading to undetectable signal. Interestingly, the M2 peptide, which have an average sensitivity of $52.7 \mathrm{mV} / \mathrm{dec}$ in PBS, shows significant sensitivity reduction in serum down to only $18 \mathrm{mV} / \mathrm{dec}$ (Figure 4B). Figure $4 \mathrm{C}$ shows matrix of Pearson correlation results between the curves obtained for different peptides in different environments. Here, we observed high correlation (>99\% confidence interval) between serum and PBS data of M0 and M1 which suggest similar activity even in different environment. On the other hand, M2 binding curve in serum is less correlated to its curve in PBS, which suggests that the binding between the peptides and T cells in a natural environment might be affected, compared to the synthetic buffer. Finally, almost no correlation was observed in $\mathbf{M} 3$ for the two samples, which suggest that the result for this peptide is mostly random noise, and the binding is undetectable.

\subsection{Cytotoxic functionality of UniCAR-T cells}

To investigate whether TMs equipped with the M0 or mutated E5B9 peptide epitope $\mathbf{M 1}$, M2 or M3 can be recognized by UniCARs and are able to redirect UniCAR-T cells to eliminate PSCAexpressing prostate cancer cells, we constructed PSCA-specific TMs differing with respect to their E5B9 sequence (M1, M2, M3) (Figure 5 A-B). These results were further compared with the TMs functionality in-vitro using PSCA-TM-M0 comprising the E5B9 M0 sequence, published elsewhere [55-57]. Firstly, binding capabilities of the novel TMs were analyzed by ELISA and flow cytometry. Figure $5 \mathrm{C}$ shows the binding curves of anti- E5B9 mAb to PSCAspecific TMs determined by ELISA. Similar to previous ELISA measurements using the E5B9 peptides, the anti- E5B9 mAb showed a binding affinity towards PSCA-TM-M0 and PSCA-TMM1 in the picomolar range, whereas towards the PSCA-TM-M2 and PSCA-TM-M3 lower binding affinity in the nanomolar range was detected. Furthermore, binding capability of the TMs to PC3 and UniCAR-T cells and the availability of the E5B9 peptide of bound TMs were analyzed by flow cytometry. As demonstrated in Figure S9, all of the TMs were able to bind their target antigen PSCA on the surface of the prostate cancer cell line PC3, confirming that in general all constructed PSCA-specific TMs possess a functional PSCA binding domain. Even more interestingly, all E5B9 peptide variants of cell-bound TMs were accessible for the anti-E5B9 mAb whereas M0 or M1 showed highest efficiency compared to M2 and M3. On the other hand, both the TM with the M0-peptide sequence and the TM with the M1-peptide sequence showed effective binding to UniCAR-T cells. However, the binding of the PSCA-TMM2 and PSCA-TM-M3 could not be detected on UniCAR-T cells via the His-tag, which could 
be most likely explained by a reduced binding affinity of the UniCAR anti-E5B9 scFv towards these mutated E5B9 variants. In agreement with the results obtained using SiNW FET and ELISA measurements, we have demonstrated that mutated E5B9 peptide epitope variants differ with respect to their availability for anti-E5B9 mAb and that the binding affinity of the antiE5B9 mAb towards E5B9 M2 and M3 was clearly reduced.

Secondly, the functionality of the novel TMs with respect to their capability to redirect UniCART cells to kill tumor cells (Figure $5 \mathrm{D}$ ) was analyzed using a chromium release assay. Therefore, UniCAR-T cells were co-cultured with PSCA-expressing prostate cancer cell line PC3 in the presence or absence of TMs. As shown in Figure $5 \mathrm{E}$, the TM with M0 or M1 peptide sequence activated UniCAR-T cells to specifically kill PSCA-expressing PC3 cells. Significant specific tumor cell lysis with high efficiency in the picomolar range (Figure $5 \mathrm{E}$ ) was measured. The killing capability of UniCAR-T cells redirected by the TM with M2 or M3 peptide sequence was considerably worse. In the presence of both TMs with either M2 or M3, no specific tumor lysis was observed. These data are in line with the previous study showing that the binding of the TMs with M2 or M3 could not be detected on UniCAR-T cells. Obviously, the affinity of the UniCAR anti-E5B9 scFv is not sufficient to appropriately bind the E5B9 peptide M2 or M3 which prevents the redirection of UniCAR-T cell against tumor cells. In agreement with SiNW FET results, we conclude that $A b$ affinity towards the TMs with the peptide mutants M2 or M3 was drastically reduced.

\section{CONCLUSION}

In summary, here we present the possibility of using SiNW FET in the development of anticancer immunotherapy using UniCAR-T cell approach for both research and clinical applications. We demonstrate that SiNW sensor chip can be applied to analyze the interaction of the anti-E5B9 mAb with the corresponding E5B9 peptide epitope. Namely, we show at the proof-of-concept level that the sensors can assist in the selection and design of the optimal UniCAR TM peptide epitope, necessary to reach a well-balanced redirection of UniCAR-T cells for an efficient tumor killing.

We observe different sensitivity to anti-E5B9 mAb detection in nanosensor functionalized with different peptide candidates and predict that the higher the sensitivity, the better binding effect to the UniCAR-T cells, and thus better tumor killing effect. Keeping that in mind, we constructed PSCA-specific TM based on representative group of E5B9 mutated variants (M1-M3) and successfully verified efficiency of these TMs. Finally, we compare our results with ELISA data which point out the outperformance of the FET devices in terms of limit of detection, working volume, measurement time and simplicity in sample preparation. Under optimal condition, the 
detection limit can go down to an attomolar level, which is around 4-5 orders of magnitude lower than LOD in ELISA. Such extreme limit of detection enables the possibility to detect low concentrated clinical specimens and, thus, simplify the pretreatment of the patient sample.

While early diagnosis impact greatly on patient survival, monitoring the patient response to the therapy is equally important, which require timely adjustment of treatment strategy [89]. In this respect, we demonstrated that SiNW FET can be applied in clinical development of the therapeutic approaches and reached the comparable sensitivity, as well as LOD, when exposed to human blood serum. This potentially extends the use of electronic nanobiosensors for monitoring UniCAR system components in patient's blood.

Finally, all achieved results makes us confident that the bionanoelectronic technology is capable to make tremendous contribution for the clinical cancer research beyond the early diagnostics by contributing in personalization and efficient monitoring of the immunotherapeutic treatment, where the quantitative analysis with the conventional methodology is not achievable.

\section{MATERIALS AND METHODS}

\subsection{Materials}

E5B9 peptide variants A0-A2 were purchased from Biosyntan LtD. Peptides M0, M1-M3 were synthesized in house as described in detail in SI 12. A phosphate-buffered saline (PBS) solution ( $\mathrm{pH}$ 7.4) was prepared from dry tablets (Sigma Aldrich, Cat\# P4417) and laboratory de-ionized water. Human blood serum is obtained from the University hospital Carl Gustav Carus Dresden.

\subsection{Device fabrication}

Si nanowire devices were fabricated by conventional top-down CMOS process on an 8 inch SOI wafer consisting of a $100 \mathrm{~nm}$ top Si layer on a $400 \mathrm{~nm}$ buried oxide (BOX) layer. The top Si layer was doped with $5 \times 10^{18} \mathrm{~cm}^{-3}$ phosphorous ions by ion implantation to make the $\mathrm{n}^{+}$channel. The active Si area including the nanowire channel region and the source/drain region was defined using a combination of a stepper and a plasma etcher. Additional phosphorus ions were implanted to the source/drain region with a concentration of over $10^{20} \mathrm{~cm}^{-3}$. The honeycomb structure of nanowire channel was formed by electron beam lithography and

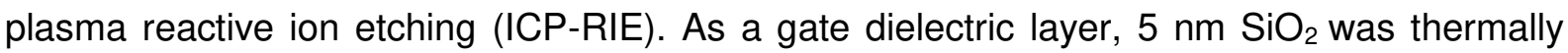
grown on the nanowire channel using a wet oxidation process. Afterwards, the source/drain electrode area was patterned by photolithography and etched with HF to remove the grown oxide layer. $100 \mathrm{~nm}$ Pt were deposited on a $20 \mathrm{~nm}$ Ti adhesion layer to form the source/drain and reference electrodes which was completed after the lift-off process. Finally, a $2 \mu \mathrm{m}$-thick 
SU-8 passivation layer was coated on the devices except for the nanowire channels and electrode pad (including the center square) region.

\subsection{Surface modification}

The SiNW surfaces were modified with respective peptides via anhydride silane conjugation followed by ethanolamine passivation (Fig.2B). Before silane deposition, SiNW sensors were cleaned with isopropanol, dried under a stream of nitrogen and placed in plasma treatment at 100W for 20 seconds. The use of 3-triethoxysilylpropyl succinic anhydride (TESPSA) for biomolecule immobilization is explained in detail in ${ }^{58}$. In short, TESPSA (Gelest Inc., USA) was left for evaporation together with the sensors in a vacuum desiccator for 4 hours under heating of IR-lamp. The sensors were then rinsed in isopropanol and dried to remove any unbound silane molecules. To completely dehydrate the surface, the sensors were cured in an oven at $120^{\circ} \mathrm{C}$ for one hour. After cooling down the sensors to room temperature, the peptides were immobilized on SiNW by placing a drop of PBS solution containing $0.1 \mathrm{mg} / \mathrm{mL}$ of peptides onto the sensor for another hour at room temperature. We used PBS and TWEEN 20 (Sigma Aldrich, Cat\# P9416) $0.05 \mathrm{v} \%$ to wash out any unbound peptide molecules. The surface was then saturated with $0.3 v \%$ ethanolaminesolution (Sigma Aldrich, Cat\# 398136) to minimize unspecific binding. We submerged the sensors in PBS for storage in a fridge at $4^{\circ} \mathrm{C}$ prior to measurement.

\subsection{SiNW FET measurement}

After removing from the storage, we rinsed the sensor with DI water and dried them under a stream of nitrogen. Different concentrations of mAb were prepared by diluting the stock with PBS solution. Starting with the lowest concentration, we incubated the sensor with a $10 \mu \mathrm{L}$ drop of solution containing mAb for 15 minutes. 0.01x PBS was used to rinse the sensor and for measurement. Diluted PBS was chosen to increase the Debye length of the sensor. A drop of $0.01 x$ PBS was placed in the center of the sensor, bridging the pseudo-reference electrode and the SiNW, while transfer characteristics of SiNW FET was captured. The electrical measurement was performed using a micro-probe-station and a source-meter-unit (Keithley 2604B) which was controlled by a Matlab program.

\subsection{ELISA test}

The analysis of the binding affinity between the E5B9 peptide derivatives and the anti-E5B9 monoclonal antibody (mAb) was tested with enzyme-linked immunosorbent assay (ELISA). ELISA test was prepared on 96-well plate (Sigma Aldrich, Cat\# A9044-2ML). Each of the peptides was tested in triplicate for each of the anti-La mAb concentrations used $(1 \mathrm{ng} / \mathrm{mL}$ to 
$5000 \mathrm{ng} / \mathrm{mL}$ ). Positive and negative controls were also performed in triplicate. After each incubation step, the wells were washed 3 times with washing buffer (PBS $+0.05 \%$ Tween 20). ELISA plates were coated with the appropriate peptide at a concentration of $10 \mathrm{ng} / \mathrm{mL}$ and incubated at $5^{\circ} \mathrm{C}$ overnight. To avoid unspecific absorption, the remaining sites were blocked with blocking solution (PBS $+5 \% \mathrm{w} / \mathrm{w}$ milk powder) for $1 \mathrm{~h}$ at room temperature. After blocking, the plates were incubated with anti-E5B9 $\mathrm{mAb}$ for $1 \mathrm{~h}$ at $37^{\circ} \mathrm{C}$. Then, HRP-coupled detection antibody (Sigma Aldrich, Cat\# CLS3590-100EA) (1:1000 v/v in PBS) was added to each well and was incubated for 30 minutes at $37^{\circ} \mathrm{C}$. TMB substrate solution was added to each well and allowed to react for $5 \mathrm{~min}$, then the reaction was stopped by adding stop solution $(0.5 \mathrm{M}$ $\mathrm{H}_{2} \mathrm{SO}_{4}$ ). The optical density was measured at $450 \mathrm{~nm}$ with an ELISA plate reader M200 (Tecan Trading AG, Switzerland).

\subsection{Data analysis}

Data analysis was performed with OriginPro 2017 software (Origin Lab Corp.). For each measurement, the transfer curve was recorded. The calibration curves were constructed by extracting the gate voltages $\left(V_{G}\right)$ that result a current equal to $10^{-7} \mathrm{~A}$ through the channel $\left(I_{s d}=10^{-}\right.$ $\left.{ }^{7} \mathrm{~A}\right)$. To make the data comparable between sensors, we normalized the data by calculating the absolute signal difference of each measurement to the value of blank sample. Michaelis Menten equation was used to fit the data and calculate dissociation constant $\mathrm{K}_{\mathrm{d}}$. We calculate the limit of detection and sensitivity of the method by linear fitting the linear range in the calibration curve in logarithm scale. To calculate the limit of detection we used the following equation $\log \left(C_{L O D}\right)=\frac{3.3 s_{B}-b}{a}=\gamma \rightarrow C_{L O D}=10^{\gamma}$ where the linear fitting is defined as $y=a x+$ $b$.

\subsection{Cytotoxicity Assay}

Cell lines. PC3 prostate cancer cell lines and 3T3 cell line were obtained from the ATCC and used without futher authentication. PC3 cell lines was transduced to express PSCA as described elsewhere [1] and was cultured in RPMI 1640 medium (Sigma Aldrich, Cat\# R088324X500ML), supplemented with $100 \mathrm{U} / \mathrm{ml}$ Penicillin/Streptomycin (Sigma Aldrich, Cat\# P0781100ML), $1 \%$ non-essential amino-acids (Sigma Aldrich, Cat\# M7145-100ML), $1 \mathrm{mM}$ Sodiumpyruvate (Biochrom, Cat\# L0473), 2 mM N-acetyl-L-alanyl-L-glutamine (Biochrom $\mathrm{GmbH}$, Cat\# K0302) and $10 \%$ FCS (Sigma Aldrich, Cat\# F0804-500ML). 3T3 cells were cultured in DMEM medium (Life Technologies, Cat\# 10569010), supplemented with $100 \mathrm{U} / \mathrm{ml}$ Penicillin/Streptomycin, $1 \%$ non-essential amino-acids and $10 \%$ FCS. All cells were kept at $37^{\circ} \mathrm{C}$ and $5 \% \mathrm{CO}_{2}$ and were cultured twice a week. 
Cloning of novel TMs. Cloning of the PSCA-TM-MO was performed as described previously [2]. For cloning of the TMs with mutated E5B9 tag (PSCA-TM-M1, PSCA-TM-M2, PSCA-TMM3), the M0 E5B9 sequence in the pSecTag2B vector encoding the PSCA-TM-M0 was replaced by dsDNA-oligos (synthesized by Eurofins Scientific SE) encoding the respective mutated E5B9 tags using the enzymes Xhol (Thermo Fisher, Cat\# ER0692) and Notl (Thermo Fisher, Cat\# ER0592). In order to establish cell lines that stably express the respective TMs, the open reading frame of the TMs was then cloned into the lentiviral p6NST50 vector using the Xbal/Nhel (Thermo Fisher, Cat\# ER0682 and Cat\# ER0972) and KspAl(Hpal)/Mssl(Pmel) (Thermo Fisher, Cat\# ER1031 and Cat\# ER1341) restriction sites.

Expression and purification of TMs. 3T3 cell lines expressing the TMs were generated with lentiviral gene transfer [3]. TMs were purified from cell culture supernatants via their $\mathrm{C}$-terminal His-tag using Ni-NTA spin columns (Qiagen GmbH, Cat\#31014). SDS-PAGE and subsequent Coomassie Brilliant Blue G250 staining [4] or Western Blot analysis [3] were used to determine TM concentration and purity.

Generation of UniCAR T cells. Buffy Coats were provided by the German Red Cross (Dresden, Germany) after obtaining consent from voluntary donors. The research with human T cells was approved by the local ethics committee of the Medical Faculty Carl Gustav Carus, Technical University Dresden (EK27022006). Peripheral blood mononuclear cells (PBMCs) were isolated from the whole blood via density gradient centrifugation using Pancoll separation solution (PanBiotech, Cat\#P04-601000). T cells were subsequently isolated from PBMCs using the human Pan T Cell Isolation Kit (Miltenyi Biotech GmbH, Cat\#130-096-535). Following isolation, $T$ cells were transduced with the UniCAR $28 / \zeta$ construct using lentiviral particles which were generated as described elsewhere [5]. For the transduction, $T$ cells were first activated using T Cell TransAct ${ }^{\mathrm{TM}}$ (Miltenyi Biotech $\mathrm{GmbH}$, Cat\#130-111-160). After $24 \mathrm{~h}, \mathrm{~T}$ cells were transduced with a multiplicity of infection (MOI) of 2 using a $2 \mathrm{~h}$ spin infection. This procedure was repeated after another $24 \mathrm{~h}$. Activation and transduction steps were performed in TexMACS ${ }^{\text {TM }}$ Medium (Miltenyi Biotech $\mathrm{GmbH}$, Cat\# 170-076-307). Transduced T cells were expanded over the weekend and used in the following week.

Flow cytometry. For binding analysis, $2 \times 10^{5}$ cells were incubated with $1 \mu \mathrm{g} / 50 \mu \mathrm{l}$ of TM for $1 \mathrm{~h}$. Binding of the TMs was detected either via their His-tag or the E5B9-tag. For the former, cells were incubated with anti-His-PE Ab (Miltenyi Biotech $\mathrm{GmbH}$, Cat\# 130-120-718) for $30 \mathrm{~min}$. For the latter, cells were incubated with $1 \mu \mathrm{g} / 50 \mu \mathrm{l}$ of the anti-La E5B9 mAb for $1 \mathrm{~h}$, followed by a 30 min incubation with Goat-anti Mouse IgG Fc Cross-Adsorbed-PE secondary ab (Thermo Fisher, Cat\# 31861). For the binding of the TMs to UniCAR T cells, $2 \times 10^{5}$ cells 
were consecutively incubated with $1 \mu \mathrm{g} / 50 \mu \mathrm{l}$ of TM for $1 \mathrm{~h}$ and anti-His-PE Ab for $30 \mathrm{~min}$. To distinguish between living and dead cells, $1 \mu \mathrm{g} / \mathrm{ml}$ popidium iodide/PBS solution (Thermo Fisher, Cat\#P3566) was added shortly before the flow cytometric measurement. For the acquisition and analysis of the data, a MACSQuant Analyzer 10 and the MACSQuantify software (Miltenyi Biotech $\mathrm{GmbH}$ ) were used.

ELISA. For ELISA measurements, the BD OptiEIA ${ }^{\mathrm{TM}}$ Reagent Set B (BD Biosciences, Cat\# 550534) was used. 96-well plates (Sigma Aldrich, Cat\# CLS3590-100EA) were coated over night with $1 \mu \mathrm{g}$ of TM per well at $4{ }^{\circ} \mathrm{C}$. After blocking, TMs were incubated with anti-La E5B9 $m A b$ in different concentrations and anti-La E5B9 mAb was subsequently detected with an anti-mouse IgG-HRP Ab (Sigma Aldrich, Cat\# A9044-2ML). After applying the substrate solution, OD at $450 \mathrm{~nm}$ was determined (NanoQuant infinite M200 Pro, Tecan Group AG) and $K_{d}$ values were calculated using GraphPad Prism 9 software (GraphPad Prism Inc.).

Chromium release assay. Killing of tumor cells with redirected UniCAR T cells was analyzed via chromium release assay as described previously [6]. Shortly, UniCAR T cells were incubated with $5 \times 10^{3}{ }^{51} \mathrm{Cr}$-labled tumor target cells at an effector-to-target cell (E:T) ratio of 5:1 with or without TMs in different concentrations. ${ }^{51} \mathrm{Cr}$ release into the co-culture supernatant was measured after $24 \mathrm{~h}$ incubation using a MicroBeta² Microplate Counter (PerkinElmer LAS $\mathrm{GmbH})$.

Statistical analysis: was performed using the GraphPad Prism 9 software (GraphPad Prism Inc.). One-way ANOVA with post hoc Dunnett multiple comparison test was used, with $p$ values below 0.0332 being considered significant $\left({ }^{*} p<0.0332\right.$, ${ }^{* *} p<0.0021,{ }^{* * *} p<0.0002$ with respect to samples w/o TM). 


\section{ASSOCIATED CONTENTS}

The Supporting Information is available free of charge at:

In-house synthesis of $\mathrm{M}$ group peptide sequences (SI1); contact angle measurement (SI2); fluorescence microscope test (SI3); Calibration curve of ELISA for 7 peptide derivatives (SI4); Pearson correlation test (SI5); Response of SiNW FET to serum sample (SI6); Comparison of BSA solution and serum (SI7); TWEEN test (SI8); Cytometry analysis of TM binding to PC3 cells, anti-La E5B9 and UniCar-T cells (SI9)

\section{AUTHOR INFORMATION:}

\section{Corresponding Authors}

Larysa Baraban - Helmholtz-Zentrum Dresden-Rossendorf e.V., Institute of Radiopharmaceutical Cancer Research, Bautzner Landstrasse 400, 01328 Dresden, Germany; https://orcid.org/0000-0003-1010-2791; Email: I.baraban@hzdr.de

Michael Bachmann -- Helmholtz-Zentrum Dresden-Rossendorf e.V., Institute of Radiopharmaceutical Cancer Research, Bautzner Landstrasse 400, 01328 Dresden, Germany; Email: m.bachmann@hzdr.de

\section{Author contribution:}

The manuscript was written through contributions of all authors. All authors have given approval to the final version of the manuscript. $¥$ These authors contributed equally.

\section{Notes}

The authors declare no competing financial interest.

\section{ACKNOWLEDGMENTS}

T.A.N.L. and L.B. acknowledges the financial support of Helmholtz Zentrum Dresden Rossendorf. L.B. thanks the German Research Foundation (grant number 413655771) for financial support. We would like to express our gratitude to Joanne K. Gross and Tim Gross 
from The Arthritis and Clinical Immunology Program, Oklahoma Medical Research Foundation and University of Oklahoma Health Sciences Center for their contribution on synthesizing and testing of the novel E5B9-peptide sequences.

The Arthritis and Clinical Immunology Program, Oklahoma Medical Research Foundation and University of Oklahoma Health Sciences Center Oklahoma City, OK 73104, USA.

\section{REFERENCES}

[1] H. Sung et al., "Global Cancer Statistics 2020: GLOBOCAN Estimates of Incidence and Mortality Worldwide for 36 Cancers in 185 Countries," CA: A Cancer Journal for Clinicians, https://doi.org/10.3322/caac. 21660 vol. 71, no. 3, pp. 209-249, 2021/05/01 2021, doi: https://doi.org/10.3322/caac.21660.

[2] M. Arruebo et al., "Assessment of the evolution of cancer treatment therapies," (in eng), Cancers (Basel), vol. 3, no. 3, pp. 3279-330, Aug 12 2011, doi: 10.3390/cancers3033279.

[3] Y. Sang, Z. Zhao, M. Zhao, P. Hao, Y. Leng, and H. Liu, "From UV to Near-Infrared, WS2 Nanosheet: A Novel Photocatalyst for Full Solar Light Spectrum Photodegradation," Advanced Materials, https://doi.org/10.1002/adma.201403264 vol. 27, no. 2, pp. 363-369, 2015/01/01 2015, doi: https://doi.org/10.1002/adma.201403264.

[4] A. K. Singh and J. P. McGuirk, "CAR T cells: continuation in a revolution of immunotherapy," (in eng), Lancet Oncol, vol. 21, no. 3, pp. e168-e178, Mar 2020, doi: 10.1016/s14702045(19)30823-x.

[5] D. A. Wall and J. Krueger, "Chimeric antigen receptor T cell therapy comes to clinical practice," (in eng), Curr Oncol, vol. 27, no. Suppl 2, pp. S115-s123, Apr 2020, doi: 10.3747/co.27.5283.

[6] C. Graham, R. Hewitson, A. Pagliuca, and R. Benjamin, "Cancer immunotherapy with CAR-T cells - behold the future," (in eng), Clin Med (Lond), vol. 18, no. 4, pp. 324-328, Aug 2018, doi: 10.7861/clinmedicine.18-4-324.

[7] C. H. June, R. S. O'Connor, O. U. Kawalekar, S. Ghassemi, and M. C. Milone, "CAR T cell immunotherapy for human cancer," (in eng), Science, vol. 359, no. 6382, pp. 1361-1365, Mar 23 2018, doi: 10.1126/science.aar6711.

[8] C. L. Bonifant, H. J. Jackson, R. J. Brentjens, and K. J. Curran, "Toxicity and management in CAR T-cell therapy," (in eng), Mol Ther Oncolytics, vol. 3, p. 16011, 2016, doi: 10.1038/mto.2016.11.

[9] M. Bachmann, "The UniCAR system: A modular CAR T cell approach to improve the safety of CAR T cells," Immunology Letters, vol. 211, pp. 13-22, 2019/07/01/ 2019, doi: https://doi.org/10.1016/j.imlet.2019.05.003.

[10] C. Arndt et al., "UniCAR T cell immunotherapy enables efficient elimination of radioresistant cancer cells," Oncolmmunology, vol. 9, no. 1, p. 1743036, 2020/01/01 2020, doi: $10.1080 / 2162402 X .2020 .1743036$.

[11] S. Loff et al., "Rapidly Switchable Universal CAR-T Cells for Treatment of CD123-Positive Leukemia," Molecular Therapy - Oncolytics, vol. 17, pp. 408-420, 2020, doi: 10.1016/j.omto.2020.04.009.

[12] L. R. Loureiro et al., "Development of a novel target module redirecting UniCAR T cells to Sialyl Tn-expressing tumor cells," (in eng), Blood cancer journal, vol. 8, no. 9, pp. 81-81, 2018, doi: 10.1038/s41408-018-0113-4.

[13] S. Albert et al., "From mono- to bivalent: improving theranostic properties of target modules for redirection of UniCAR T cells against EGFR-expressing tumor cells in vitro and in vivo," (in eng), Oncotarget, vol. 9, no. 39, pp. 25597-25616, 2018, doi: 10.18632/oncotarget.25390. 
[14] S. Albert et al., "A novel nanobody-based target module for retargeting of T lymphocytes to EGFR-expressing cancer cells via the modular UniCAR platform," Oncolmmunology, vol. 6, no. 4, p. e1287246, 2017/04/03 2017, doi: 10.1080/2162402X.2017.1287246.

[15] M. Wermke et al., "Proof-of-concept for Rapidly Switchable Universal CAR-T Platform with UniCAR-T-CD123 in Relapsed/Refractory AML," Blood, 2021, doi:

10.1182/blood.2020009759.

[16] B. Ibarlucea et al., "Ultrasensitive detection of Ebola matrix protein in a memristor mode," Nano Research, vol. 11, no. 2, pp. 1057-1068, 2018/02/01 2018, doi: 10.1007/s12274-0171720-2.

[17] S. Klinghammer et al., "Nanosensor-Based Real-Time Monitoring of Stress Biomarkers in Human Saliva Using a Portable Measurement System," ACS Sensors, 2020/12/03 2020, doi: 10.1021/acssensors.0c02267.

[18] K. Kim, M.-J. Kim, D. W. Kim, S. Y. Kim, S. Park, and C. B. Park, "Clinically accurate diagnosis of Alzheimer's disease via multiplexed sensing of core biomarkers in human plasma," Nature Communications, vol. 11, no. 1, p. 119, 2020/01/08 2020, doi: 10.1038/s41467-019-13901-z.

[19] S. Xu et al., "Real-time reliable determination of binding kinetics of DNA hybridization using a multi-channel graphene biosensor," Nature Communications, vol. 8, no. 1, p. 14902, 2017/03/21 2017, doi: 10.1038/ncomms14902.

[20] M. T. Hwang et al., "Ultrasensitive detection of nucleic acids using deformed graphene channel field effect biosensors," Nature Communications, vol. 11, no. 1, p. 1543, 2020/03/24 2020, doi: 10.1038/s41467-020-15330-9.

[21] N. T. Tung et al., "Peptide aptamer-modified single-walled carbon nanotube-based transistors for high-performance biosensors," Scientific Reports, vol. 7, 12/20 2017, doi: 10.1038/s41598-017-18169-1.

[22] D. Rani et al., "Top-Down Fabricated Silicon Nanowire Arrays for Field-Effect Detection of Prostate-Specific Antigen," ACS Omega, vol. 3, no. 8, pp. 8471-8482, 2018/08/31 2018, doi: 10.1021/acsomega.8b00990.

[23] P. Zhang et al., "Electrochemically Exfoliated High-Quality 2H-MoS2 for Multiflake Thin Film Flexible Biosensors," Small, https://doi.org/10.1002/smll.201901265 vol. 15, no. 23, p. 1901265, 2019/06/01 2019, doi: https://doi.org/10.1002/smll.201901265.

[24] J. Lee et al., "Two-dimensional Layered MoS2 Biosensors Enable Highly Sensitive Detection of Biomolecules," Scientific Reports, vol. 4, no. 1, p. 7352, 2014/12/17 2014, doi: 10.1038/srep07352.

[25] J. Liu et al., "Ultrasensitive Monolayer MoS2 Field-Effect Transistor Based DNA Sensors for Screening of Down Syndrome," Nano Letters, vol. 19, no. 3, pp. 1437-1444, 2019/03/13 2019, doi: 10.1021/acs.nanolett.8b03818.

[26] Y. Liang et al., "Wafer-Scale Uniform Carbon Nanotube Transistors for Ultrasensitive and Label-Free Detection of Disease Biomarkers," ACS Nano, vol. 14, no. 7, pp. 8866-8874, 2020/07/28 2020, doi: 10.1021/acsnano.0c03523.

[27] J. Schütt et al., "Nanocytometer for smart analysis of peripheral blood and acute myeloid leukemia: a pilot study," Nano Letters, vol. 20, no. 9, pp. 6572-6581, 2020/09/09 2020, doi: 10.1021/acs.nanolett.0c02300.

[28] L. F. E. Huerta-Nuñez et al., "A biosensor capable of identifying low quantities of breast cancer cells by electrical impedance spectroscopy," Scientific Reports, vol. 9, no. 1, p. 6419, 2019/04/23 2019, doi: 10.1038/s41598-019-42776-9.

[29] F. Khosravi et al., "Static micro-array isolation, dynamic time series classification, capture and enumeration of spiked breast cancer cells in blood: the nanotube-CTC chip," (in eng), Nanotechnology, vol. 27, no. 44, pp. 44LT03-44LT03, 2016, doi: 10.1088/09574484/27/44/44LT03.

[30] D. P. Tran et al., "Toward Intraoperative Detection of Disseminated Tumor Cells in Lymph Nodes with Silicon Nanowire Field Effect Transistors," ACS Nano, vol. 10, no. 2, pp. 23572364, 2016/02/23 2016, doi: 10.1021/acsnano.5b07136. 
[31] K. Ditte et al., "Ultrasoft and High-Mobility Block Copolymers for Skin-Compatible Electronics," Advanced Materials, https://doi.org/10.1002/adma.202005416 vol. 33, no. 4, p. 2005416, 2021/01/01 2021, doi: https://doi.org/10.1002/adma.202005416.

[32] D. Blasi et al., "Printed, cost-effective and stable poly(3-hexylthiophene) electrolyte-gated field-effect transistors," Journal of Materials Chemistry C, 10.1039/D0TC03342A vol. 8, no. 43, pp. 15312-15321, 2020, doi: 10.1039/D0TC03342A.

[33] C.-S. Lee, S. K. Kim, and M. Kim, "Ion-sensitive field-effect transistor for biological sensing," (in eng), Sensors (Basel, Switzerland), vol. 9, no. 9, pp. 7111-7131, 2009, doi: 10.3390/s90907111.

[34] N. Rohaizad, C. C. Mayorga-Martinez, Z. Sofer, and M. Pumera, "1T-Phase Transition Metal Dichalcogenides (MoS2, MoSe2, WS2, and WSe2) with Fast Heterogeneous Electron Transfer: Application on Second-Generation Enzyme-Based Biosensor," ACS Applied Materials \& Interfaces, vol. 9, no. 46, pp. 40697-40706, 2017/11/22 2017, doi: 10.1021/acsami.7b13090.

[35] J. Pas et al., "Neurospheres on Patterned PEDOT:PSS Microelectrode Arrays Enhance Electrophysiology Recordings," Advanced Biosystems, vol. 2, 12/01 2017, doi: 10.1002/adbi.201700164.

[36] X. Y. Wong, A. Sena-Torralba, R. Álvarez-Diduk, K. Muthoosamy, and A. Merkoçi, "Nanomaterials for Nanotheranostics: Tuning Their Properties According to Disease Needs," ACS Nano, vol. 14, no. 3, pp. 2585-2627, 2020/03/24 2020, doi: 10.1021/acsnano.9b08133.

[37] D. A. Koutsouras et al., "Simultaneous monitoring of single cell and of micro-organ activity by PEDOT:PSS covered multi-electrode arrays," Materials Science and Engineering: $C$, vol. 81, pp. 84-89, 2017/12/01/2017, doi: https://doi.org/10.1016/j.msec.2017.07.028.

[38] J. Liu et al., "Syringe-injectable electronics," Nature Nanotechnology, vol. 10, no. 7, pp. 629636, 2015/07/01 2015, doi: 10.1038/nnano.2015.115.

[39] R. Liu et al., "High Density Individually Addressable Nanowire Arrays Record Intracellular Activity from Primary Rodent and Human Stem Cell Derived Neurons," Nano Letters, vol. 17, no. 5, pp. 2757-2764, 2017/05/10 2017, doi: 10.1021/acs.nanolett.6b04752.

[40] D. Kuzum et al., "Transparent and flexible low noise graphene electrodes for simultaneous electrophysiology and neuroimaging," Nature Communications, vol. 5, no. 1, p. 5259, 2014/10/20 2014, doi: 10.1038/ncomms6259.

[41] I. Nardi-Agmon et al., "Exhaled Breath Analysis for Monitoring Response to Treatment in Advanced Lung Cancer," Journal of Thoracic Oncology, vol. 11, no. 6, pp. 827-837, Jun 2016, doi: 10.1016/j.jtho.2016.02.017.

[42] Z. Hao, Y. Pan, W. Shao, Q. Lin, and X. Zhao, "Graphene-based fully integrated portable nanosensing system for on-line detection of cytokine biomarkers in saliva," Biosensors \& Bioelectronics, vol. 134, pp. 16-23, Jun 1 2019, doi: 10.1016/j.bios.2019.03.053.

[43] M. Tertis, P. I. Leva, D. Bogdan, M. Suciu, F. Graur, and C. Cristea, "Impedimetric aptasensor for the label-free and selective detection of Interleukin-6 for colorectal cancer screening," Biosensors \& Bioelectronics, vol. 137, pp. 123-132, Jul 15 2019, doi: 10.1016/j.bios.2019.05.012.

[44] B. Viet Anh Pham et al., "Modified Floating Electrode-Based Sensors for the Quantitative Monitoring of Drug Effects on Cytokine Levels Related with Inflammatory Bowel Diseases," Acs Applied Materials \& Interfaces, vol. 10, no. 20, pp. 17100-17106, May 23 2018, doi: 10.1021/acsami.8b04287.

[45] R. Elshafey, A. C. Tavares, M. Siaj, and M. Zourob, "Electrochemical impedance immunosensor based on gold nanoparticles-protein $\mathrm{G}$ for the detection of cancer marker epidermal growth factor receptor in human plasma and brain tissue," Biosensors \& Bioelectronics, vol. 50, pp. 143-149, Dec 15 2013, doi: 10.1016/j.bios.2013.05.063.

[46] E. Arkan, R. Saber, Z. Karimi, and M. Shamsipur, "A novel antibody-antigen based impedimetric immunosensor for low level detection of HER2 in serum samples of breast cancer patients via modification of a gold nanoparticles decorated multiwall carbon 
nanotube-ionic liquid electrode," Analytica Chimica Acta, vol. 874, pp. 66-74, May 18 2015, doi: 10.1016/j.aca.2015.03.022.

[47] J. W. Kamande et al., "Modular Microsystem for the Isolation, Enumeration, and Phenotyping of Circulating Tumor Cells in Patients with Pancreatic Cancer," Analytical Chemistry, vol. 85, no. 19, pp. 9092-9100, Oct 1 2013, doi: 10.1021/ac401720k.

[48] D. P. Tran et al., "Toward Intraoperative Detection of Disseminated Tumor Cells in Lymph Nodes with Silicon Nanowire Field Effect Transistors," Acs Nano, vol. 10, no. 2, pp. 23572364, Feb 2016, doi: 10.1021/acsnano.5b07136.

[49] Y. Yang, Y. Fu, H. Su, L. Mao, and M. Chen, "Sensitive detection of MCF-7 human breast cancer cells by using a novel DNA-labeled sandwich electrochemical biosensor," Biosensors \& Bioelectronics, vol. 122, pp. 175-182, Dec 30 2018, doi: 10.1016/j.bios.2018.09.062.

[50] A. Benvidi and S. Jahanbani, "Self-assembled monolayer of SH-DNA strand on a magnetic bar carbon paste electrode modified with Fe304@Ag nanoparticles for detection of breast cancer mutation," Journal of Electroanalytical Chemistry, vol. 768, pp. 47-54, May 12016 , doi: 10.1016/j.jelechem.2016.02.038.

[51] H. Qiu, A. Sarathy, K. Schulten, and J.-P. Leburton, "Detection and mapping of DNA methylation with 2D material nanopores," Npj 2d Materials and Applications, vol. 1, Apr 11 2017, doi: 10.1038/s41699-017-0005-7.

[52] A. Bonanni, I. Fernandez-Cuesta, X. Borrise, F. Perez-Murano, S. Alegret, and M. del Valle, "DNA hybridization detection by electrochemical impedance spectroscopy using interdigitated gold nanoelectrodes," Microchimica Acta, vol. 170, no. 3-4, pp. 275-281, Sep 2010, doi: 10.1007/s00604-010-0358-5.

[53] M. L. Coluccio et al., "Detection of single amino acid mutation in human breast cancer by disordered plasmonic self-similar chain," Science Advances, vol. 1, no. 8, Sep 2015, Art no. e1500487, doi: 10.1126/sciadv.1500487.

[54] H. Abiri et al., "Monitoring the spreading stage of lung cells by silicon nanowire electrical cell impedance sensor for cancer detection purposes," Biosensors \& Bioelectronics, vol. 68, pp. 577-585, Jun 15 2015, doi: 10.1016/j.bios.2015.01.057.

[55] M. Abdolahad, H. Shashaani, M. Janmaleki, and S. Mohajerzadeh, "Silicon nanograss based impedance biosensor for label free detection of rare metastatic cells among primary cancerous colon cells, suitable for more accurate cancer staging," Biosensors \& Bioelectronics, vol. 59, pp. 151-159, Sep 15 2014, doi: 10.1016/j.bios.2014.02.079.

[56] S. D. Bukkitgar and N. P. Shetti, "Electrochemical Sensor for the Determination of Anticancer Drug 5-Fluorouracil at Glucose Modified Electrode," Chemistryselect, vol. 1, no. 4, pp. 771777, Apr 2016, doi: 10.1002/slct.201600197.

[57] R. Karthik et al., "A facile graphene oxide based sensor for electrochemical detection of prostate anti-cancer (anti-testosterone) drug flutamide in biological samples," Rsc Advances, vol. 7, no. 41, pp. 25702-25709, 2017 2017, doi: 10.1039/c6ra28792a.

[58] B. P. Timko, T. Cohen-Karni, Q. Qing, B. Tian, and C. M. Lieber, "Design and Implementation of Functional Nanoelectronic Interfaces With Biomolecules, Cells, and Tissue Using Nanowire Device Arrays," leee Transactions on Nanotechnology, vol. 9, no. 3, pp. 269-280, May 2010, doi: 10.1109/tnano.2009.2031807.

[59] N. Tien Anh, T.-I. Yin, D. Reyes, and G. A. Urban, "Microfluidic Chip with Integrated Electrical Cell-Impedance Sensing for Monitoring Single Cancer Cell Migration in Three-Dimensional Matrixes," Analytical Chemistry, vol. 85, no. 22, pp. 11068-11076, Nov 19 2013, doi: 10.1021/ac402761s.

[60] C. Laborde et al., "Real-time imaging of microparticles and living cells with CMOS nanocapacitor arrays," Nature Nanotechnology, vol. 10, no. 9, pp. 791-+, Sep 2015, doi: 10.1038/nnano.2015.163.

[61] Y. Zhang et al., "Spearhead Nanometric Field-Effect Transistor Sensors for Single-Cell Analysis," Acs Nano, vol. 10, no. 3, pp. 3214-3221, Mar 2016, doi: 10.1021/acsnano.5b05211. 
[62] H.-P. Peng et al., "Label-free electrochemical DNA biosensor for rapid detection of mutidrug resistance gene based on Au nanoparticles/toluidine blue-graphene oxide nanocomposites," Sensors and Actuators B-Chemical, vol. 207, pp. 269-276, Feb 2015, doi: 10.1016/j.snb.2014.10.059.

[63] X. Wu et al., "Selective determination of drug resistant cancer cells on indium tin oxide electrode modified with nano titanium dioxide," Electrochemistry Communications, vol. 12, no. 7, pp. 962-965, Jul 2010, doi: 10.1016/j.elecom.2010.05.002.

[64] P. Ambhorkar et al., "Nanowire-Based Biosensors: From Growth to Applications," (in eng), Micromachines, vol. 9, no. 12, p. 679, 2018, doi: 10.3390/mi9120679.

[65] D. P. Tran, T. T. T. Pham, B. Wolfrum, A. Offenhäusser, and B. Thierry, "CMOS-Compatible Silicon Nanowire Field-Effect Transistor Biosensor: Technology Development toward Commercialization," Materials, vol. 11, no. 785, 5, 2018.

[66] G. Zheng, F. Patolsky, Y. Cui, W. U. Wang, and C. M. Lieber, "Multiplexed electrical detection of cancer markers with nanowire sensor arrays," Nature Biotechnology, vol. 23, no. 10, pp. 1294-1301, 2005/10/01 2005, doi: 10.1038/nbt1138.

[67] F. Patolsky, G. Zheng, and C. M. Lieber, "Fabrication of silicon nanowire devices for ultrasensitive, label-free, real-time detection of biological and chemical species," Nature Protocols, vol. 1, no. 4, pp. 1711-1724, 2006/11/01 2006, doi: 10.1038/nprot.2006.227.

[68] E. Baek et al., "Negative Photoconductance in Heavily Doped Si Nanowire Field-Effect Transistors," Nano Letters, vol. 17, no. 11, pp. 6727-6734, 2017/11/08 2017, doi: 10.1021/acs.nanolett.7b02788.

[69] E. Baek et al., "Intrinsic plasticity of silicon nanowire neurotransistors for dynamic memory and learning functions," Nature Electronics, vol. 3, no. 7, pp. 398-408, 2020/07/01 2020, doi: 10.1038/s41928-020-0412-1.

[70] T. Rim, C.-K. Baek, K. Kim, Y.-H. Jeong, j.-s. Lee, and M. Meyyappan, "Silicon Nanowire Biologically Sensitive Field Effect Transistors: Electrical Characteristics and Applications," Journal of nanoscience and nanotechnology, vol. 14, pp. 273-87, 04/15 2014, doi: 10.1166/jnn.2014.8760.

[71] B. Ibarlucea, T. Rim, C. K. Baek, J. A. G. M. de Visser, L. Baraban, and G. Cuniberti, "Nanowire sensors monitor bacterial growth kinetics and response to antibiotics," Lab on a Chip, 10.1039/C7LC00807D vol. 17, no. 24, pp. 4283-4293, 2017, doi: 10.1039/C7LC00807D.

[72] K. Kim, T. Rim, C. Park, D. Kim, M. Meyyappan, and J.-S. Lee, "Suspended honeycomb nanowire ISFETs for improved stiction-free performance," Nanotechnology, vol. 25, no. 34, p. 345501, 2014/08/05 2014, doi: 10.1088/0957-4484/25/34/345501.

[73] T. Rim, M. Meyyappan, and C.-K. Baek, "Optimized operation of silicon nanowire field effect transistor sensors," (in eng), Nanotechnology, vol. 25, no. 50, p. 505501, 2014/12// 2014, doi: 10.1088/0957-4484/25/50/505501.

[74] B. Ibarlucea et al., "Gating Hysteresis as an Indicator for Silicon Nanowire FET Biosensors," Applied Sciences, vol. 8, no. 6, 2018, doi: 10.3390/app8060950.

[75] C.-A. Vu, W.-P. Hu, Y.-S. Yang, H. W.-H. Chan, and W.-Y. Chen, "Signal Enhancement of Silicon Nanowire Field-Effect Transistor Immunosensors by RNA Aptamer," ACS Omega, vol. 4, no. 12, pp. 14765-14771, 2019/09/17 2019, doi: 10.1021/acsomega.9b01264.

[76] J. Lee et al., "A Highly Responsive Silicon Nanowire/Amplifier MOSFET Hybrid Biosensor," Scientific Reports, vol. 5, no. 1, p. 12286, 2015/07/21 2015, doi: 10.1038/srep12286.

[77] C. Arndt et al., "Redirection of CD4+ and CD8+ T lymphocytes via a novel antibody-based modular targeting system triggers efficient killing of PSCA+ prostate tumor cells," (in eng), Prostate, vol. 74, no. 13, pp. 1347-58, Sep 2014, doi: 10.1002/pros.22851.

[78] A. Feldmann et al., "Retargeting of T lymphocytes to PSCA- or PSMA positive prostate cancer cells using the novel modular chimeric antigen receptor platform technology "UniCAR"," Oncotarget; Vol 8, No 19, 2017. [Online]. Available: https://www.oncotarget.com/article/15572/text/. 
[79] A. B. Raff, A. Gray, and W. M. Kast, "Prostate stem cell antigen: a prospective therapeutic and diagnostic target," (in eng), Cancer letters, vol. 277, no. 2, pp. 126-132, 2009, doi: 10.1016/j.canlet.2008.08.034.

[80] A. Feldmann, C. Arndt, S. Koristka, N. Berndt, R. Bergmann, and M. P. Bachmann, "Conventional CARs versus modular CARs," (in eng), Cancer immunology, immunotherapy : Cll, vol. 68, no. 10, pp. 1713-1719, 2019, doi: 10.1007/s00262-019-02399-5.

[81] C. Arndt et al., "A theranostic PSMA ligand for PET imaging and retargeting of T cells expressing the universal chimeric antigen receptor UniCAR," (in eng), Oncoimmunology, vol. 8, no. 11, pp. 1659095-1659095, 2019, doi: 10.1080/2162402X.2019.1659095.

[82] A. Gang, G. Gabernet Garriga, L. Renner, L. Baraban, and G. Cuniberti, "A simple two-step silane-based (bio-) receptor molecule immobilization without additional binding site passivation," RSC Adv., vol. 5, 04/13 2015, doi: 10.1039/C5RA04469C.

[83] Y. Henchoz, J. Schappler, L. Geiser, J. Prat, P. A. Carrupt, and J. L. Veuthey, "Rapid determination of pK (a) values of 20 amino acids by CZE with UV and capacitively coupled contactless conductivity detections," (in eng), Anal Bioanal Chem, vol. 389, no. 6, pp. 186978, Nov 2007, doi: 10.1007/s00216-007-1568-5.

[84] M. Kuchar, M. Pretze, T. Kniess, J. Steinbach, J. Pietzsch, and R. Löser, "Site-selective radiolabeling of peptides by (18)F-fluorobenzoylation with [(18F)]SFB in solution and on solid phase: a comparative study," (in eng), Amino Acids, vol. 43, no. 4, pp. 1431-43, Oct 2012, doi: 10.1007/s00726-012-1216-z.

[85] C.-H. Chu et al., "Beyond the Debye length in high ionic strength solution: direct protein detection with field-effect transistors (FETs) in human serum," Scientific Reports, vol. 7, no. 1, p. 5256, 2017/07/12 2017, doi: 10.1038/s41598-017-05426-6.

[86] N. Lloret et al., "Effects of buffer composition and dilution on nanowire field-effect biosensors," (in eng), Nanotechnology, vol. 24, no. 3, p. 035501, Jan 25 2013, doi: 10.1088/0957-4484/24/3/035501.

[87] H. P. Erickson, "Size and shape of protein molecules at the nanometer level determined by sedimentation, gel filtration, and electron microscopy," (in eng), Biological procedures online, vol. 11, pp. 32-51, 2009, doi: 10.1007/s12575-009-9008-x.

[88] M. Leeman, J. Choi, S. Hansson, M. U. Storm, and L. Nilsson, "Proteins and antibodies in serum, plasma, and whole blood-size characterization using asymmetrical flow field-flow fractionation (AF4)," (in eng), Analytical and bioanalytical chemistry, vol. 410, no. 20, pp. 4867-4873, 2018, doi: 10.1007/s00216-018-1127-2. 


\section{Figures}

A

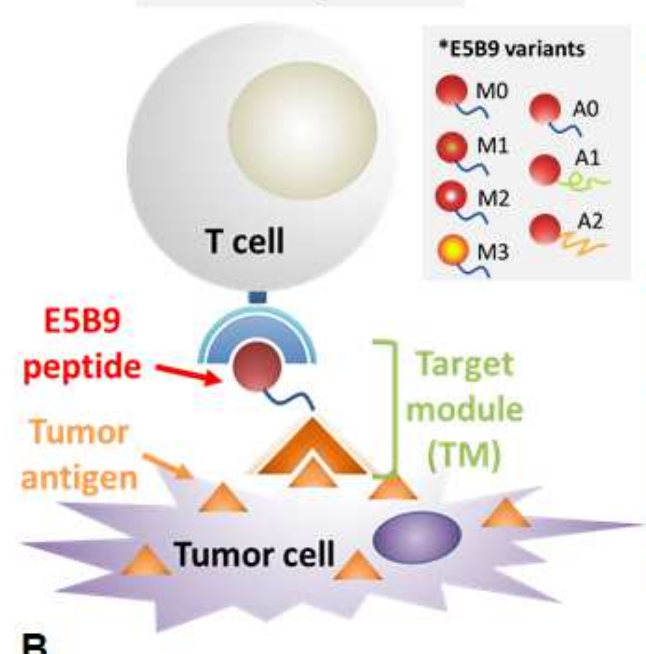

B

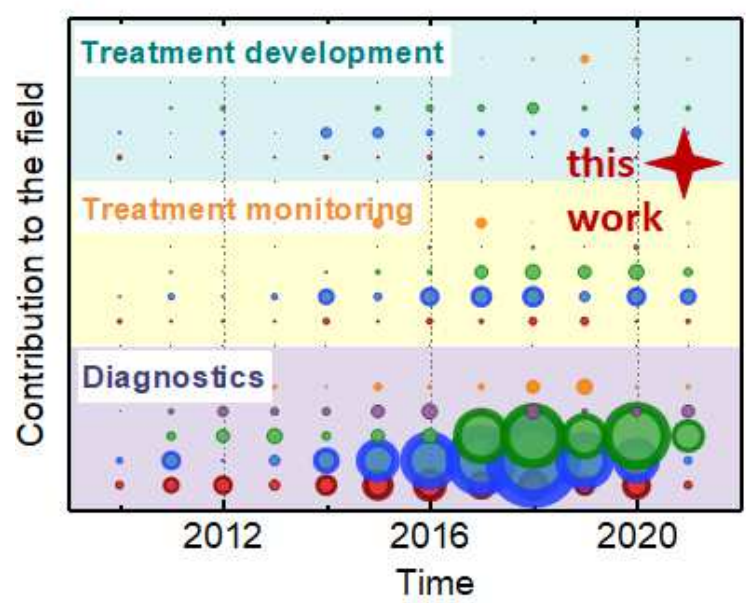

D

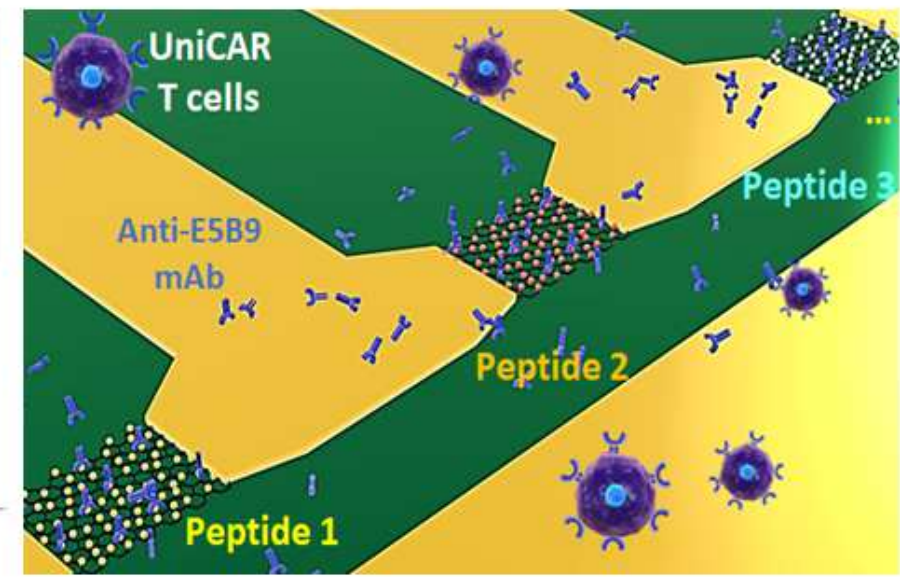

C

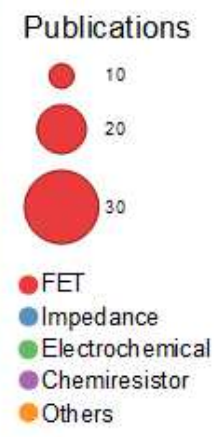

\begin{tabular}{|l|}
\hline M0 \\
\hline M1 \\
\hline M2 \\
\hline M0 \\
\hline A1 \\
\hline A2 \\
\hline
\end{tabular}

CSKPLPEVTDEY-CONH ${ }_{2}$ CSKPLPEVTGEY-CONH ${ }_{2}$ CSKPLPEVTGGY-CONH ${ }_{2}$ CSKPLPGVTDGY-CONH SKPLPEVTDEY-OH SKPLPEVTDEY-PEG ${ }_{2}-$ $\mathrm{PEG}_{2}-\mathrm{NH}-\left(\mathrm{CH}_{2}\right)_{2}-\mathrm{SH}$ Mpr-PEG $-\mathrm{PEG}_{2}-$ SKPLPEVTDEY-OH

Figure 1: (A) Concept of UniCAR-T cell therapy approach: The target module (TM) consists of an anti-tumor binding domain (orange) equipped with the peptide sequence E5B9 (red) that is recognized by UniCAR T-cell. UniCAR-T cells are only activated after their cross-linkage with tumor cell by the TMs. (B) 10-year research landscape of nano-electronic application in cancer research. The color of the circle indicates the main method that was applied in the study while the radius represents number of publications released each year in the relevant topic. (C) Library of seven variants of the E5B9 peptide as candidates for constructing TMs in UniCAR system. The red color highlights the components differ from the original E5B9 sequence. (D) Concept of the present work: different peptide variants were immobilized on active region of SiNW FETs and used to sense against anti-E5B9 mAb to screen for most effective peptide. 
A
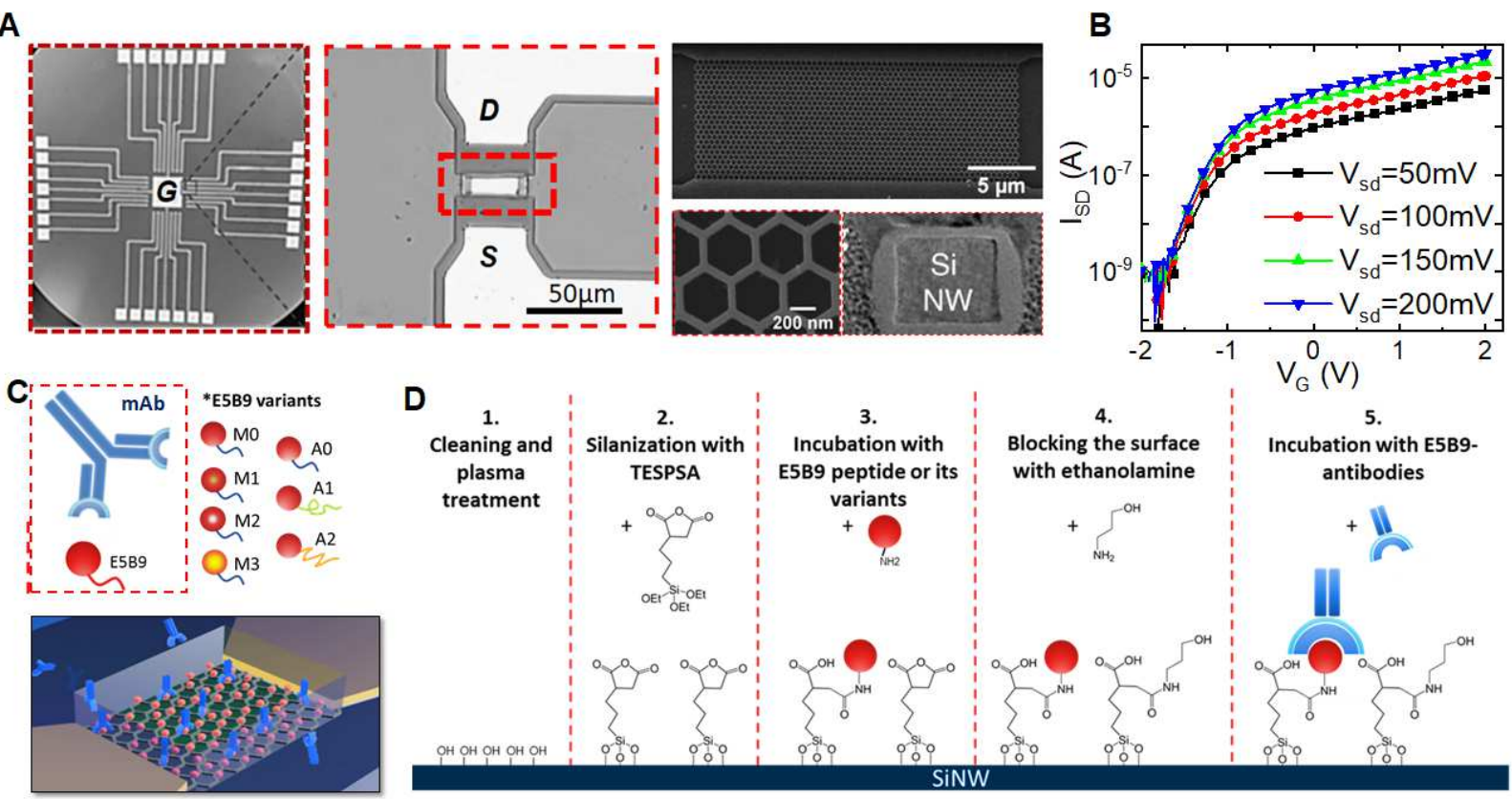

5.

4.

Blocking the surface Incubation with E5B9with ethanolamine $+\int^{\mathrm{OH}} \quad+\mathbb{1}$

(0)

(1)
(3)
(4)

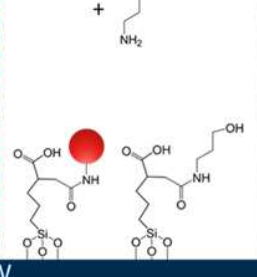

G

(2)

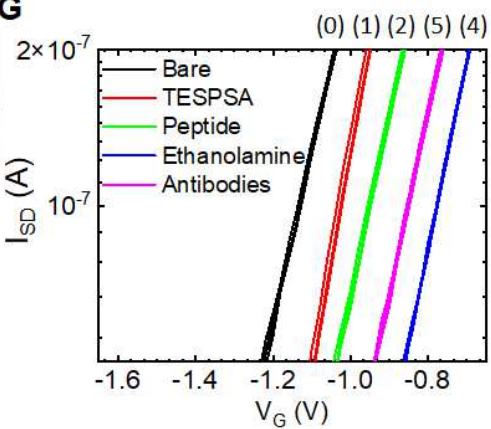

TESPSA + Lift off

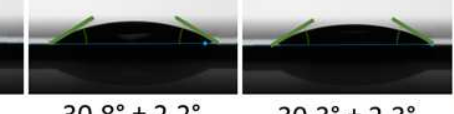

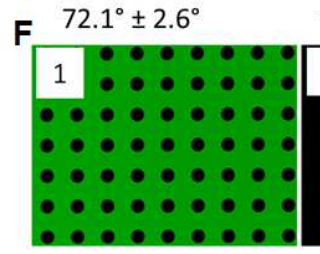

Resist pattern

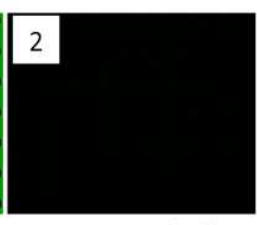

Peptide immobilization E5B9-Ab + 2nd Fluor. Ab

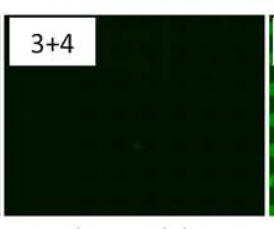

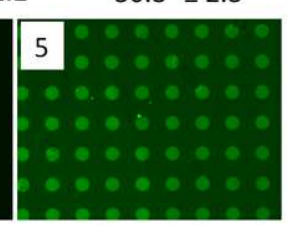

Figure 2: (A) Overview of our SiNW FET sensor: microscopic image and SEM images of a chip containing 16 SiNW pair electrodes and a square reference electrode in the center. $(B)$ Electrical characterization of a typical SiNW FET shows that the device has n-type characteristics and an 'ON' state even at $V_{G}=0 \mathrm{~V}$. (C) Illustration of anti-E5B9 mAb testing with different E5B9 variants. (D) Sensor surface functionalization process: (1) introduction of hydroxyl group on bare SiNW surface by plasma cleaning; (2) creation of a monolayer of TESPSA onto SiNW through evaporation in vacuum desiccator; (3) immobilization of peptides onto the surface by reaction of amino-group forming amide bond; (4) saturation of the surface to reduce non-specific binding with ethanolamine and (5) incubation with anti-E5B9 mAb solution for 15 minutes before measurement. (E) Contact angle measurement of a bare silicon substrate after each functionalization step. (F) Fluorescence microscopy image showing a glass slide covered with photoresist hole-pattern and then followed the described functionalization steps. Note that the photoresist was removed after silanization process and fluorescence signal in the last image was mostly from the Alexa Fluor $488 \mathrm{lg}$ G secondary Abs bound to the anti-E5B9 mAb. (G) Measurement of transfer curve of the SiNW FET after each step of modification. Note that all the tests (E-G) were done with MO E5B9 peptide. 
A
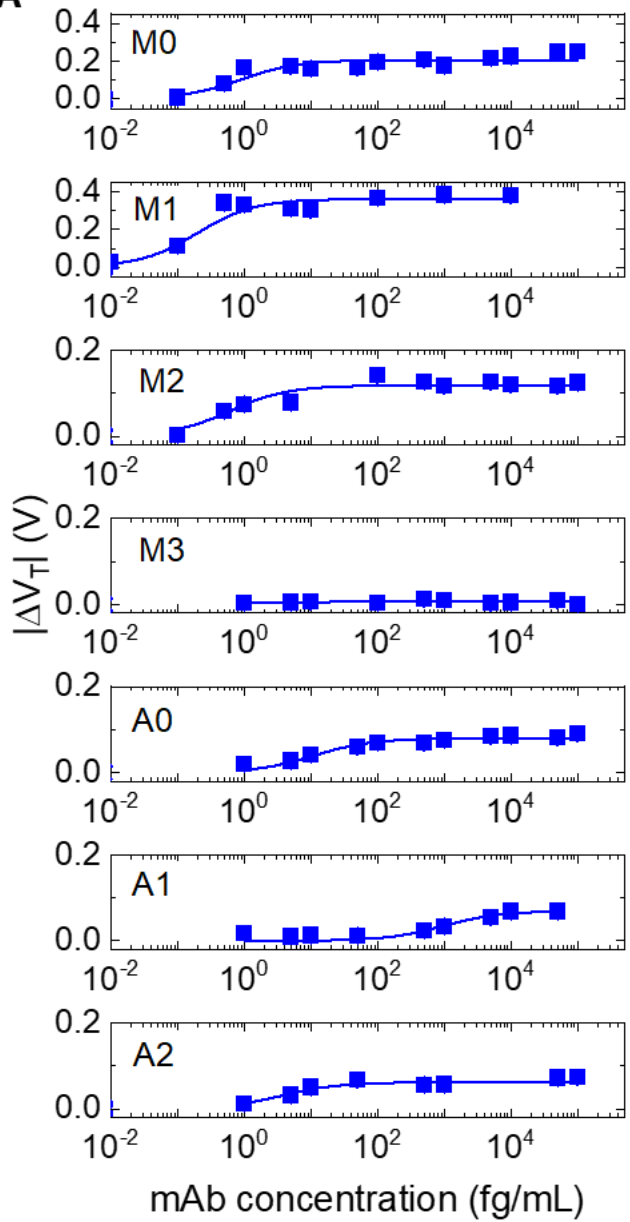

B

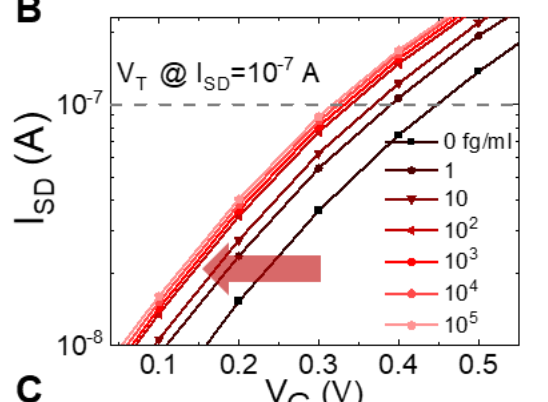

C
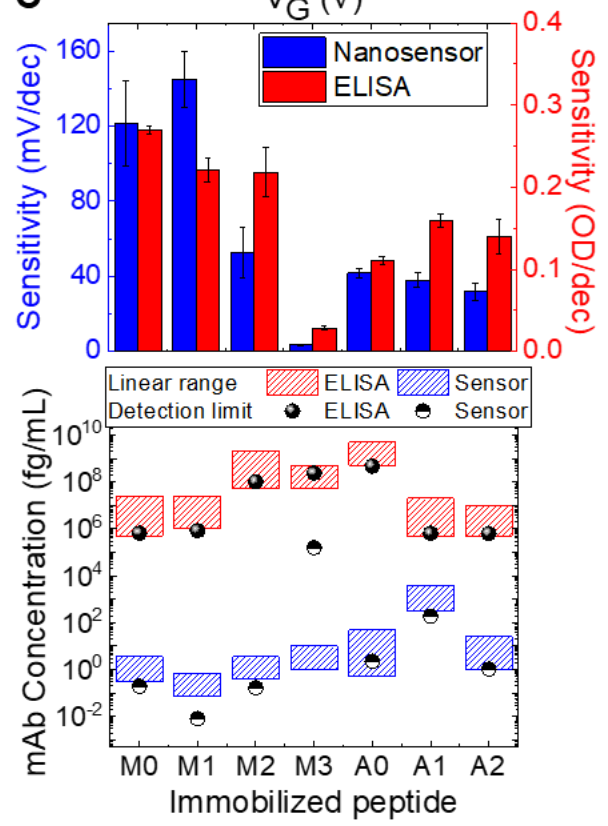

Figure 3: (A) Calibration of anti-E5B9 Abs by SiNW FET with different immobilized E5B9 peptides. The data were normalized relatively to the blank sample measured on each sensor and fitted using Michaelis Menten function. (B) Exemplary transfer characteristics of $\boldsymbol{A O}$ functionalized SiNW FET measured at $V_{S D}=0.1 \mathrm{~V}$ in $0.01 \mathrm{x} P B S$ after incubation with increasing concentration of mAb in $1 x$ PBS. (C) Comparison of sensor characteristics between SiNW FET and ELISA method. Upper panel presents the sensitivity of each measurement. Lower panel illustrates limit of detection (circle) and linear range (bars) of SiNW FET (blue) and ELISA (red). 
Table 1: Summary of test performance during titration of mAb to immobilized peptides in SiNW FET and ELISA method. Sensitivities are given in $m V$ and $O D$ per decade of concentration.

\begin{tabular}{|l|c|c|c|c|c|}
\hline & $\begin{array}{c}\text { Sensitivity } \\
\text { (mV/decade) }\end{array}$ & \multicolumn{1}{c}{ LOD (M) } & \multicolumn{1}{c|}{$\boldsymbol{K}_{\mathbf{d}}(\mathbf{M})$} & $\begin{array}{c}\text { ELISA } \\
\text { Sensitivity } \\
\text { (OD/dec) }\end{array}$ & \multicolumn{1}{|c|}{ LOD (M) } \\
\hline M0 & $121.5 \pm 22.8$ & $1.28 \times 10^{-18}$ & $3.40 \times 10^{-11}$ & $0.270 \pm 0.005$ & $4.32 \times 10^{-12}$ \\
\hline M1 & $145.0 \pm 15.0$ & $5.38 \times 10^{-20}$ & $3.49 \times 10^{-11}$ & $0.220 \pm 0.015$ & $5.54 \times 10^{-12}$ \\
\hline M2 & $52.7 \pm 13.3$ & $1.09 \times 10^{-18}$ & $3.12 \times 10^{-9}$ & $0.218 \pm 0.030$ & $6.77 \times 10^{-10}$ \\
\hline M3 & $3.4 \pm 0.3$ & $1.05 \times 10^{-12}$ & $1.47 \times 10^{-9}$ & $0.028 \pm 0.003$ & $1.58 \times 10^{-9}$ \\
\hline A0 & $41.8 \pm 2.5$ & $1.50 \times 10^{-17}$ & $2.00 \times 10^{-8}$ & $0.110 \pm 0.005$ & $3.16 \times 10^{-9}$ \\
\hline A1 & $38.0 \pm 3.7$ & $1.25 \times 10^{-15}$ & $2.23 \times 10^{-11}$ & $0.160 \pm 0.008$ & $4.24 \times 10^{-12}$ \\
\hline A2 & $31.8 \pm 4.8$ & $6.92 \times 10^{-18}$ & $1.89 \times 10^{-11}$ & $0.140 \pm 0.021$ & $4.19 \times 10^{-12}$ \\
\hline
\end{tabular}


A
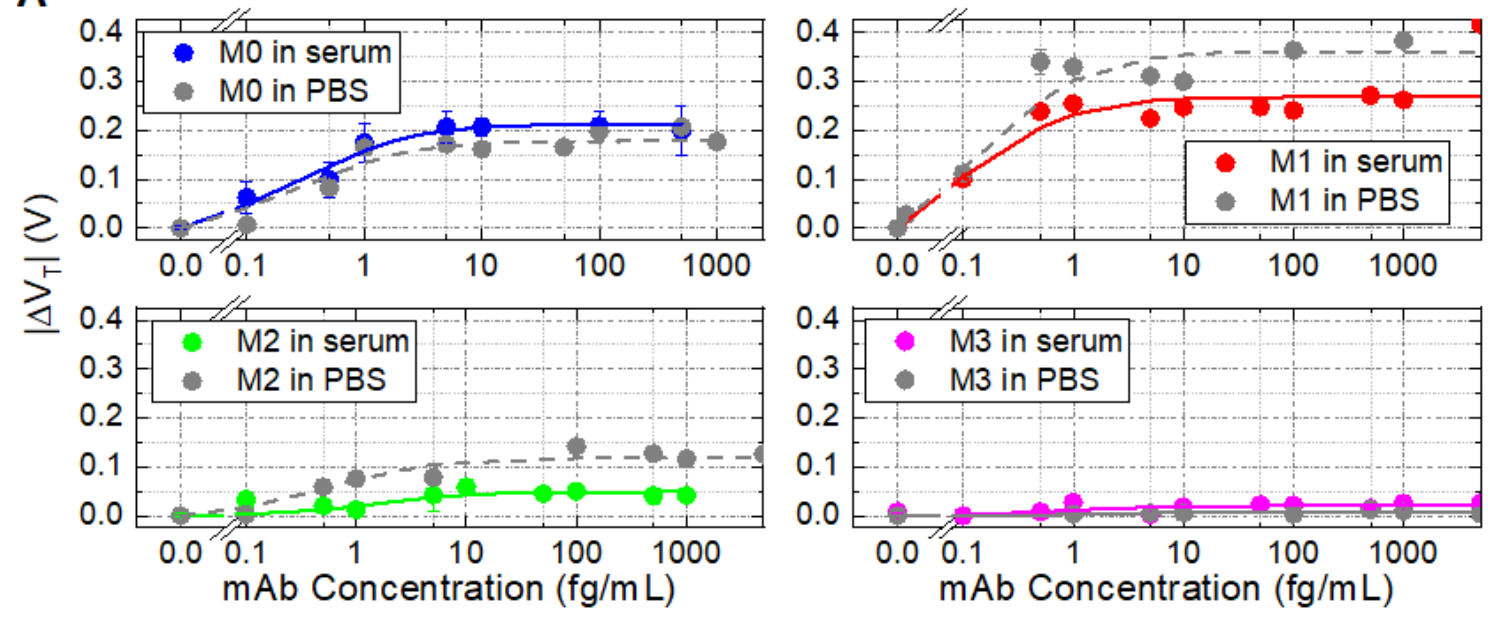

B

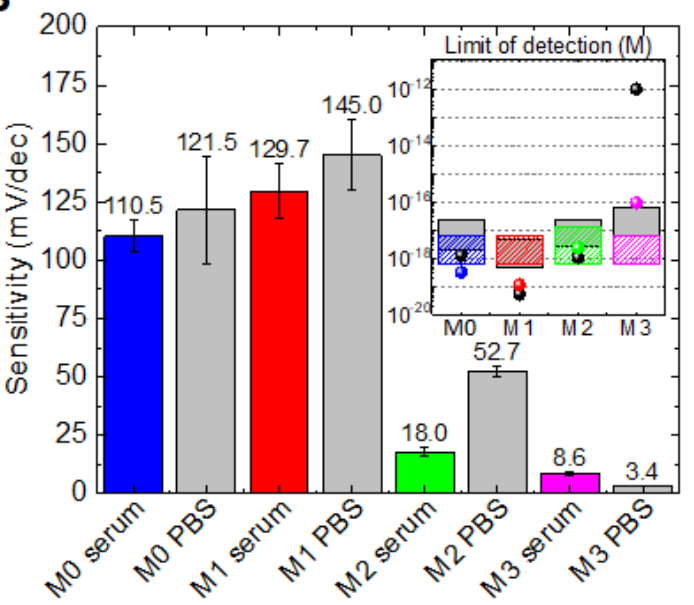

C $\quad{ }^{*} p<=0.05^{* *} p<=0.01^{* * *} p<=0.001$

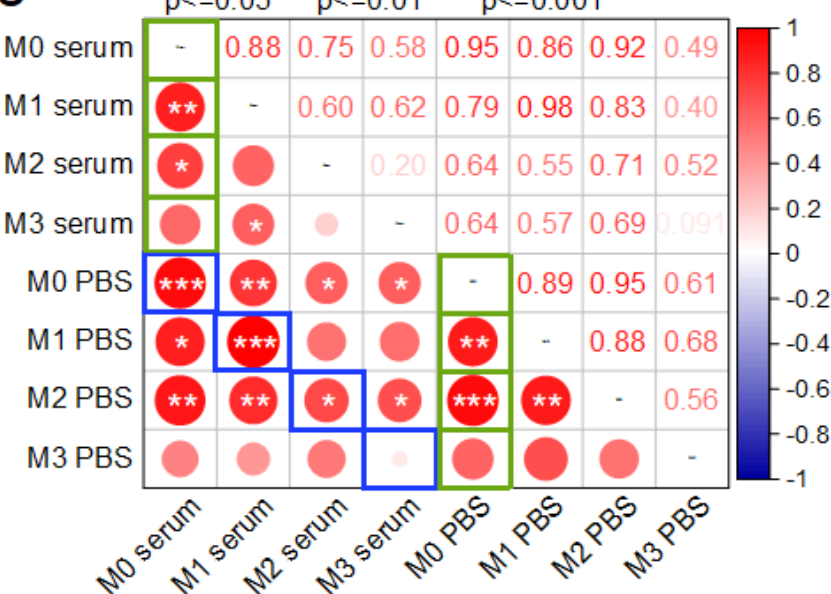

Figure 4: Result of measurement conducted in human blood serum. (A) Calibration of anti$5 B 9$ Abs in PBS (gray, dash line) and in serum (color, solid line) on different peptides; (B) comparison of sensor performance between tests done in PBS (gray) and serum (color) (inset: illustration of linear range (bars) and limit of detection (squares) of each measurement); (C) Matrix shows Pearson correlation coefficients and significant levels between titration curves of different E5B9-peptide variants in different samples. The closer the coefficient to 1 or -1 , the stronger the correlation between that data. The blue boxes highlight the comparison results between PBS and serum sample, while green boxes feature comparison between wild type E5B9 peptide to other peptide variants in the same samples (serum/PBS). 
A

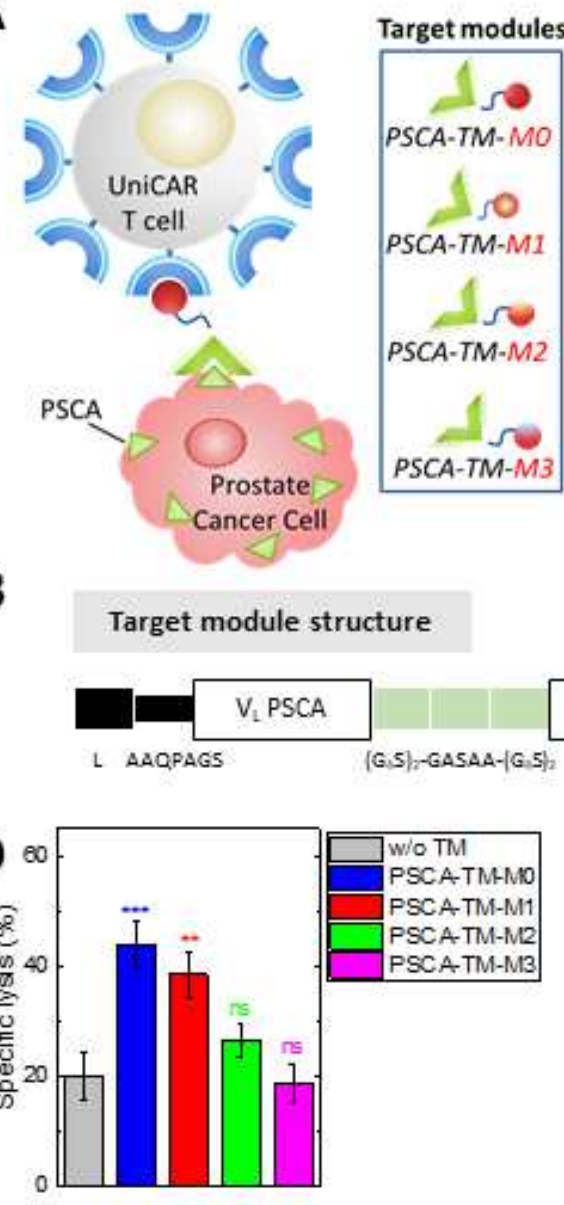

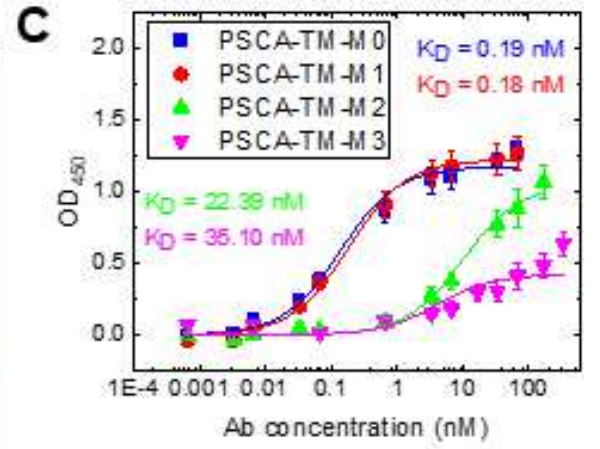

MO: SKPLPEVTDEY

M1: SKPLPEVTGEY

M2: SKPLPEVTGGY M3: SKPLPGVTDGY

\section{$V_{H}$ PSCA $\square 5 B 9$}

ARGGP myc his:

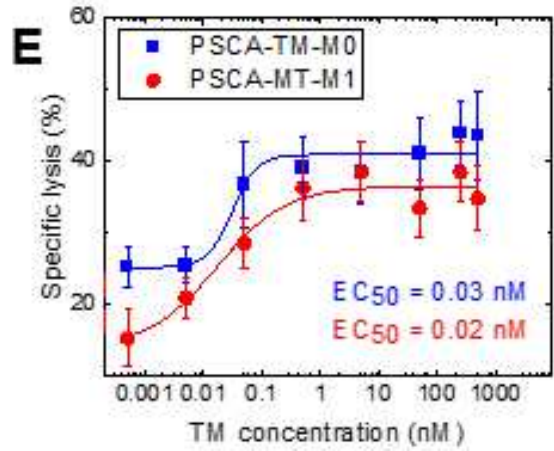

Figure 5: (A) Schematic illustration of UniCAR-T cells cross-linked to prostate cancer cells via an anti-PSCA target module (TM) equipped with different E5B9 peptide variants. (B) Construction of the wildtype PSCA-TM-MO and the novel PSCA-TMs with mutated E5B9 peptide epitope (M1-M3). (C) Binding analysis by ELISA. ELISA-plates were coated with $1 \mu \mathrm{g}$ of TM per well and incubated with different concentrations of the anti-E5B9 mAb. Binding was detected with anti-mouse IgG-HRP through measuring of $O D_{450}$. Mean of $O D_{450}+/-S E M$ for three independent experiments is shown. $(D, E)$ In a chromium release killing assay, UniCAR$T$ cells were incubated with ${ }^{51} \mathrm{Cr}$-labeled PC3-PSCA tumor cells at a ratio of 5:1 either in the absence (w/O) or (D) presence of $250 \mathrm{nM}$ PSCA-TM or $(E)$ in the presence of different concentrations of the indicated PSCA-TMs. ${ }^{51} \mathrm{Cr}$ release was measured after $24 \mathrm{~h}$ incubation. In the diagrams, mean specific lysis +/- SEM for three independent experiments with three different $T$ cell donors is shown. $\left({ }^{*} p<0.0332,{ }^{* *} p<0.0021,{ }^{* *} p<0.0002\right.$ with respect to samples w/o TM, One-way-ANOVA followed by Dunnett's post hoc test). (M0: E5B9 wild type, M1-M3: E5B9 mutant variants). 


\section{Supplementary Files}

This is a list of supplementary files associated with this preprint. Click to download.

- SINguyenle.pdf 